\title{
Memory Effects in Population Dynamics: Spread of Infectious Disease as a Case Study
}

\author{
A. Pimenov ${ }^{1}$, T.C. Kelly ${ }^{2}$, A. Korobeinikov ${ }^{3 *}$, M.J.A. O'Callaghan ${ }^{4}$, A.V. Pokrovskii ${ }^{4}$, \\ D. Rachinskii ${ }^{4}$ \\ ${ }^{1}$ Weierstrass Institute, Mohrenstrasse 39, D-10117 Berlin, Germany \\ 2 Department of Biology, Earth and Environmental Science, University College Cork, Ireland \\ ${ }^{3}$ MACSI, Department of Mathematics and Statistics, University of Limerick, Ireland \\ ${ }^{4}$ Department of Applied Mathematics, University College Cork, Ireland
}

\begin{abstract}
Modification of behaviour in response to changes in the environment or ambient conditions, based on memory, is typical of the human and, possibly, many animal species. One obvious example of such adaptivity is, for instance, switching to a safer behaviour when in danger, from either a predator or an infectious disease. In human society such switching to safe behaviour is particularly apparent during epidemics. Mathematically, such changes of behaviour in response to changes in the ambient conditions can be described by models involving switching. In most cases, this switching is assumed to depend on the system state, and thus it disregards the history and, therefore, memory. Memory can be introduced into a mathematical model using a phenomenon known as hysteresis. We illustrate this idea using a simple SIR compartmental model that is applicable in epidemiology. Our goal is to show why and how hysteresis can arise in such a model, and how it may be applied to describe a variety of memory effects. Our other objective is to introduce a unified paradigm for mathematical modelling with memory effects in epidemiology and ecology. Our approach treats changing behaviour as an irreversible flow related to large ensembles of elementary exchange operations that recently has been successfully applied in a number of other areas, such as terrestrial hydrology, and macroeconomics. For the purposes of illustrating these ideas in an application to biology, we consider a rather simple case study and develop a model from first principles. We accompany the model with extensive numerical simulations which exhibit interesting qualitative effects.
\end{abstract}

Keywords and phrases: mathematical epidemiology, SIR model, hysteresis, PETS, adaptation, memory effects, equilibrium, infectious disease, Preisach operator, operator-differential equations, dynamics, public information, olfactory

Mathematics Subject Classification: 92D30, 47J40

\section{Introduction}

The ability to respond optimally to changing conditions is an essential feature of life (e.g. [65]). Changing behaviour in response to changes in the environment is particularly apparent in the event of epidemics.

\footnotetext{
*Corresponding author. E-mail: andrei.korobeinikov@ul.ie
} 
A. Pimenov, T.C. Kelly, A. Korobeinikov, M.J.A. O’Callaghan, A.V. Pokrovskii, D. Rachinskii Memory effects in population dynamics

Intelligent responses, organised or spontaneous, such as isolation of infected individuals in order to prevent the spread of disease, were practised and recorded fairly early in history. Probably the earliest recorded evidence of such practice is given in Leviticus Chapter 13 of the Old Testament:

"If the shiny spot on the skin is white but does not appear to be more than skin deep and the hair in it has not turned white, the priest is to isolate the affected person for seven days. On the seventh day the priest is to examine them, and if he sees that the sore is unchanged and has not spread in the skin, he is to isolate them for another seven days." ( $L v .13: 4-5)$.

Memory, inter alia, enables the prediction of the future on the basis of experience, and thus it is a vital component of the mechanism of survival and reproductive success and, therefore, of fitness. Memory, in some form, may be ubiquitous throughout the biological realm. "Implicit memory" is found among heterotrophic eukaryotes, e.g. mollusks and insects, and is classified into short-, medium- and longterm forms [38]. Considerable advances in the understanding of both the physiological and molecular mechanisms involved in implicit memory have been made [38,43]. A map-like spatial memory, which is used in visual navigation by insects (e.g. bees and ants), has been discovered [18,63]. Explicit, or declarative memory, which characterises sentient man, evolved from the ancestral implicit state [43]. However, there is mounting evidence that a sophisticated "episodic-like" memory is possessed by some birds, e.g. crows (Corvidae) $[17,27,28]$, which enables them to remember "when, where and what" in relation to past events, and to plan for the future, in effect to engage in mental time travel - a property previously thought to be associated exclusively with Homo sapiens [71]. Currently, the interpretation of some of these findings in relation to memory in non-human animals have proved controversial (e.g. [39]; but see also $[10,59])$ and is still being debated $[9,25,37]$.

The development of vaccines identified a powerful capacity for long-term memory in the adaptive immune response [3,78]. A remarkable example of this is provided by [77] who showed that neutralising antibodies derived from B cells of survivors of the 1918 Spanish flu pandemic were still effective some 90 years later. The surviving individuals were aged between 91 and 101 (that is they were aged between 2 and 12 in 1918) and had apparently retained adaptive immunity over the interval. Therefore an understanding of psychological, neuroecological and immunological memory has considerable practical implications. "Novel" emerging pathogens are widely perceived to be among the major risks posed to modern society because of the absence of herd immunity. Likewise, "novel" predators, which have either been accidentally or deliberately introduced to geographical areas from which they were previously absent, have inflicted considerable damage and extinction of the native fauna precisely because of their naivete and inability to recognise the invaders as predators $[7,20,24]$. Recent insights into horizontal gene transfer (HGT) between prokaryotes [46,47], and the role of viruses, lead Goldenfeld and Woese [35] to state that "viruses are an important repository and memory of a community's genetic information". Viruses are now considered to have evolved very early in the history of life on Earth [33], which suggests that a capacity for memory is part of an ancient lineage within the biological realm. However, the mechanisms involved in memory storage which involves a "Dialogue between genes and synapses" [43] are complex at both the molecular $[57,60]$ and cellular levels of organisation $[26,79]$.

An effect of memory is that temporary stimuli can cause permanent effects upon human or animal behaviour. Such Permanent Effects of a Temporary Stimulus (PETS) can be mathematically described by the term "hysteresis". An aim of this paper is to demonstrate how both the ability to respond to a threat, and memory, can affect the dynamics of a biosystem, and to introduce a unified paradigm for PETS mathematical modelling in biology. To do this, we apply an approach which is based on concepts used in modelling irreversible flows related to large ensembles of elementary exchange operations, which recently has been applied successfully in such areas as terrestrial hydrology [4], and macroeconomics [21, 22]. The general theory of ordinary differential equations with Preisach model, which we apply to an epidemic model in this paper, is well understood and reliable numerical solution methods have been developed $[55,56]$.

Here we use a basic Susceptible, Infectious, Recovered (SIR) epidemic model as a convenient example of a system where these ideas can be readily applied. Using this type of framework, we derive, from first 
A. Pimenov, T.C. Kelly, A. Korobeinikov, M.J.A. O’Callaghan, A.V. Pokrovskii, D. Rachinskii Memory effects in population dynamics

principles, a model which incorporates memory. As a test bed, we compare behaviour of the SIR model without temporary stimuli, which is well studied, cf. $[2,6,14]$, with its counterpart in a population that is strongly affected by the aforementioned stimuli. The dynamics of the latter model exhibit remarkable qualitative effects, which we illustrate by extensive numerical simulation.

We use the SIR model as a convenient example to demonstrate the ideas and methods of PETS modelling. It is obvious, however, that this approach can be applied to a much wider range of models and to similar phenomena that arise in epidemiology, population dynamics and other areas of mathematical biology. The exposition will be organized as follows. In Section 2, we briefly recall relevant facts and ideas from classical mathematical epidemiology and introduce the SIR model. In Section 3 we identify where and how the relationships describing PETS should be included in such models. However, the rigourous description of these relationships are not specified at this stage but is deferred to Section 4 . This description is based on the classical Preisach operator which is a cornerstone of the modern mathematical theory of hysteresis (see [62] and further references therein). In Section 4 we also provide results of numerical computational experiments, which exhibit remarkable qualitative effects, and we discuss methods of qualitative analysis of the class of operator-differential equations which we introduced.

\section{SIR model of infectious disease}

\subsection{The basic SIR model}

To describe the spread of an infectious disease in a population, Kermack and McKendrick [45] developed in 1927 a compartmental model that is known as a SIR (or Susceptible-Infectious-Recovered) model. The ideas which were employed in this model proved to be highly effective for studying the spread of infectious diseases [44] and served as a basis for developing more sophisticated models that were successfully applied to real-life situations, such as the spread of Foot and Mouth Disease [31], Severe Acute Respiratory Syndrome (SARS) [58], West Nile virus [76], as well as many other infections. According to the assumptions of Kermack and McKendrick [45], the population is divided into three distinct classes of epidemiological significance, namely the susceptible subpopulation, the infectious subpopulation and the recovered (or removed) subpopulation; we denote by $S(t), I(t)$ and $R(t)$ the fractions of the susceptible, infectious and removed individuals in the population, respectively. A susceptible individual can be infected through contact with an infectious individual, and immediately after acquiring the infection the newly infected individual moves into the infectious compartment and, subsequently, as a result of recovery or isolation, into the removed class. If the recovered individuals retain their immunity permanently, then they remain in the recovered compartment. The transfer diagram for this model is

$$
S \longrightarrow I \longrightarrow R
$$

It is commonly assumed that there is a continuous influx with a constant rate $b$ of individuals into the population due to birth and recruitment. All newborns are assumed healthy and susceptible, and hence enter the susceptible compartment. Another traditional assumption is that an infection is transmitted via direct contacts between the infectious and the susceptibles (so-called horizontal transmission) and, according to the principle of mass action [44], occurs with a rate that is bilinear with respect to (i.e. directly proportional to the product of) the number of susceptible and infectious individuals, $\beta S I$. Under these assumptions, the model can be described by the following system of ordinary differential equations:

$$
\begin{aligned}
\dot{S} & =b-\beta S I-\mu S, \\
\dot{I} & =\beta S I-(\gamma+\mu+\sigma) I, \\
\dot{R} & =\gamma I-\mu R .
\end{aligned}
$$


A. Pimenov, T.C. Kelly, A. Korobeinikov, M.J.A. O’Callaghan, A.V. Pokrovskii, D. Rachinskii Memory effects in population dynamics

Here, a dot denotes the time derivative, $b$ is the birth or recruitment rate, $\mu$ is the rate of natural mortality, $\sigma$ is the rate of mortality inflicted by the disease, $\gamma$ is the rate of recovery and $\beta$ is the incidence rate. All these coefficients are strictly positive. For the sake of simplicity, here we assumed that the natural deaths from all three compartments occur at the same rate $\mu$; this assumption is not very restrictive, and all the results and methods can be immediately extended to a model in which the death rates for all compartments are different. It is easy to see that mathematically the third equation for $R(t)$ is decoupled from the first and second equations, and hence it can be omitted, and the system can be reduced to a 2-dimensional system for $S(t)$ and $I(t)$.

As we mentioned, the properties of this model are well studied $[2,6,14]$. This model always has an infection-free equilibrium state in which $I=0$, and the entire population is healthy and susceptible. The system can also have an endemic equilibrium state, where $S, I>0$, and hence the infection persists endemically. The existence of the endemic equilibrium state and the overall properties of the system depend on the so-called basic reproduction number of a pathogen [40], which in this case is

$$
R_{0}=b \beta / \mu(\gamma+\mu+\sigma) .
$$

The basic reproduction number $R_{0}$ of a disease has a transparent biological meaning: it is the number of secondary cases produced by a single infected individual introduced into an entirely susceptible population. It may be expected from this definition that the infection persists when $R_{0}>1$ and dies out when $R_{0} \leq 1$. Computations and analysis support this deduction: it was demonstrated and rigorously proven (cf. e.g. [14]) that for $R_{0}>1$ there exists an endemic equilibrium state, which is globally asymptotically stable. The global asymptotic stability means, in this case, that the system eventually approaches the endemic infective level and maintains this constant level indefinitely. In contrast, for $R_{0} \leq 1$, the system has no positive equilibria, and the infection-free equilibrium is globally asymptotically stable. This implies that, for any non-negative initial condition, the infection should eventually disappear from the population.

\subsubsection{An example}

For the purpose of illustration, we describe a simple example. Consider a densely populated university campus. The population of such habitat is typically of order of 10,000 individuals, which is large enough to apply the SIR approach. Of course, the campus is not an isolated habitat; however in the epidemiological context the contacts within the campus p lay the major role for its inhabitants. Mortality, as such, is negligible for this particular example; births and mortality should be replaced respectively by recruitment and departure. The students remain on campus for 4-5 years, and hence the coefficients $b$ and $\mu$ are of the order 0.1 year $^{-1}$ (typically close to 0.2 year $^{-1}$ ). We can consider the spread of influenza, for instance H1N1 [34], in this campus. In this case $\sigma$ is negligible, and the coefficient $\beta$ may be estimated to be of order of $10^{2}$ year $^{-1}$. The latent period (during which an individual is infected but not yet infectious) for influenza is very short and is of the order of 0.0001 year, and can be neglected. Thus, the usage of the SIR model is justified. We have to note, however, that there is a strong seasonality in this particular example, as a university campus is generally much less populated in summer than in autumn/winter/spring.

\subsection{Adapting behaviour to the conditions: Safe and Risky modes of behaviour}

Due to its simplicity, the SIR model described in the previous section assumes that the hosts are not able to respond in any way to the advent of an epidemic and disregards the ability of the community to adapt its behaviour to the danger. Such an assumption is probably justified for an animal population: even if an animal is able to recognise the threat of infection, we hardly can expect that it is able to work out any protective measures. There is some speculation that it may however initiate avoidance procedures in response to Public Information [23]. Humans, however, are able to intelligently respond: in response to a threat, an individual can change its usual behaviour for a "safer mode of behaviour". People receive and perceive information regarding the epidemic from the "outside" world, and they can adjust their behaviour to avoid or to reduce the risk of being infected. Typical aspects of such a "safer behaviour" 
A. Pimenov, T.C. Kelly, A. Korobeinikov, M.J.A. O’Callaghan, A.V. Pokrovskii, D. Rachinskii Memory effects in population dynamics

may include simple precautionary measures, such as refraining from potentially dangerous contacts, using vaccination in a case of influenza, using protection (condoms) in a case of sexually transmitted diseases, taking an extra portion of vitamin $\mathrm{C}$ in the case of a common cold, or simply adjusting a general life style. The number of individuals who exhibit the safer behaviour is, in turn, affected by some aggregated temporary stimuli, such as awareness in the population about the current severity of the epidemic, the availability of effective and inexpensive medicines, media and/or administrative pressure, etc.

The information that can influence the shift in the behaviour comes from a variety of sources, which can be classified into two major categories:

1. Personal observation: a person can see people around him/her being infected, or hear from other people about the danger of infection (including unprompted rumours, i.e. rumours that spread without any government control).

2. Mass media, including any kind of messages spread by reporters, health officials, or volunteers.

We note, as above, that in animal populations there may be a Public Information system [23] which alerts, at least, a proportion of a local animal population to a growing epidemic. It is thus possible our model may be extended to include animal populations. The principal difference between the two categories above is that the first is not fully controllable. Furthermore, in this scenario individuals become aware of the danger when infection comes to their immediate neighbourhood and when the number of infecteds is evidently increasing. In contrast, the second category can be completely controllable by the authorities. If we assume that the effort for spreading information through mass media is driven by the amount of financial resources (money) invested, then we can explore the efficacy of different strategies. We consider separately the case where information comes from the first category of sources in the absence of mass media (for instance, the infection spreads locally). Then we treat the situation where the information comes exclusively from the sources identified in the second category in the absence of the first category, an assumption justified, for example, for infections with a long period of latency, such as HIV or tuberculosis.

Our aim is to construct a compartmental model which incorporates the possibility of shifting the behaviour to safe mode. We assume that individuals have knowledge about the symptoms and possible outcomes of an infection, and hence they are able to recognise that the infection is spreading and comprehend the danger. We also assume that the population is aware of the measures that enhance safety. For the sake of simplicity, we assume that each individual can choose between one of two possible distinctive modes of behaviour, namely Safe and Risky behaviours, and that the susceptible population $S$ is accordingly divided into two groups,

$$
S=S_{S} \cup S_{R}
$$

Here and later, as they occur in subscripts, $S$ stands for Safe and $R$ for Risky. We denote by $P_{S}(t)$ and $P_{R}(t)$ the fractions of individuals who adopt Safe and Risky modes of behaviour, respectively, assuming that these fractions vary in time in response to change in epidemic conditions. In particular,

$$
P_{S}(t)+P_{R}(t)=1 .
$$

In the SIR model framework, the difference between these two modes is that they have different incidence rates, $\beta_{S}$ and $\beta_{R}$, respectively, such that $\beta_{S}<\beta_{R}$ holds. Under these assumptions, the equations of the SIR model are

$$
\begin{aligned}
& \dot{S}=b-\left(\beta_{R} P_{R}+\beta_{S} P_{S}\right) S I-\mu S, \\
& \dot{I}=\left(\beta_{R} P_{R}+\beta_{S} P_{S}\right) S I-(\gamma+\mu+\sigma) I,
\end{aligned}
$$

which, by (2.4), can be rewritten as

$$
\begin{aligned}
& \dot{S}=b-\left(\beta_{R}\left(1-P_{S}\right)+\beta_{S} P_{S}\right) S I-\mu S, \\
& \dot{I}=\left(\beta_{R}\left(1-P_{S}\right)+\beta_{S} P_{S}\right) S I-(\gamma+\mu+\sigma) I .
\end{aligned}
$$


A. Pimenov, T.C. Kelly, A. Korobeinikov, M.J.A. O’Callaghan, A.V. Pokrovskii, D. Rachinskii Memory effects in population dynamics

This system is not closed now because we added the variable $P_{S}(t)$ which must be defined: we should add an equation that describes the evolution of $P_{S}$ in time. We assume that $P_{S}(t)$ is related in some way to an external pressure, $A(t)$, which we term the Influence Function (or Pressure), and which measures the aggregated stimuli to change an individual's behaviour to Safe mode. The simplest assumption is to postulate that $P_{S}(t)$ is a function of $A(t)$. However, this assumption implies that memory effects, which are the motivation of this paper, are completely disregarded. Therefore we assume, instead, a more general relation where the fraction $P_{S}(\tau)$ at a particular instant $\tau$ is determined by the history of the Influence Function or aggregated stimuli $A(t)$ before $\tau$. In mathematical terms it means that, rather than there be a functional relationship between $P_{S}(\tau)$ and $A(t)$, there exists a history/memory operator $W$ that relates the output $P_{S}(t)$ to the input $A(t)$ :

$$
P_{S}(t)=(W A)(t) .
$$

Our goal now is to introduce an adequate mathematical relationship between the aggregated stimuli or pressure $A(t)$ to switch to Safe mode and the proportion $P_{S}(t)$ of those exhibiting Safe mode of behaviour. This is the principal objective of the next section.

\section{Modelling relationships between stimulus and behaviour}

\subsection{Memory component}

We supposed above that there exists an Influence Function $A(t)$ which measures the aggregated stimuli and that this can pressurise individuals to consider changes in their behaviour in respect to their safety. We will return to particular ideas about measuring $A(t)$ later; here we simply assume that $A(t)$ is a given input, and our goal is to express $P_{S}(t)$ in terms of $A(t)$.

The simplest approach would be to assume that $A(t)$ is proportional to the prevalence of the pathogen in the population, i.e. $A(t)=\alpha I$. Then we can express $P_{S}(t)$ as an explicit function of the number of infecteds. This assumption corresponds to the first types of stimuli (see Section 2.2) and leads to a model with a nonlinear incidence rate,

$$
\begin{aligned}
& \dot{S}=b-g(I) S-\mu S, \\
& \dot{I}=g(I) S-(\gamma+\mu+\sigma) I .
\end{aligned}
$$

Here the specific form of function $g(I)$ is determined by the particularities of functional dependence of $A(t)$ on $I(t)$ and $P_{S}(t)$ on $A(t)$. If the shifting to Safe mode is promoted by an increase in the number of infected, then the probability of transmission per capita of the infectious will decrease with the increase in the number of infectious; that is, $\frac{d^{2} g}{d I^{2}} \leq 0$ should hold.

Clearly the simple linear relation $A(t)=\alpha I$ can be developed to incorporate a dependence of $A(t)$ on $S(t)$ as well. However, neither assumption involves a principal change in the dynamics of the system. Disregarding particular forms of functional dependence of $A(t)$ on $I(t)$ and $S(t)$, and of $P_{S}(t)$ on $A(t)$, the outcome of these assumptions is a nonlinear incidence term $f(S, I)$, and hence we can rewrite the model in the general form:

$$
\begin{aligned}
\dot{S} & =b-f(S, I)-\mu S, \\
\dot{I} & =f(S, I)-(\gamma+\mu+\sigma) I .
\end{aligned}
$$

Extending the condition on $g(I)$ earlier, the function $f(S, I)$ should satisfy $\frac{\partial^{2} f}{\partial I^{2}} \leq 0$. This property will be relied on in further analysis.

Both of these models were comprehensively investigated recently [48-51,53], and their properties were established. It was rigorously proved that the condition $\frac{\partial^{2} f}{\partial I^{2}} \leq 0$ is sufficient to ensure the global stability 
A. Pimenov, T.C. Kelly, A. Korobeinikov, M.J.A. O’Callaghan, A.V. Pokrovskii, D. Rachinskii Memory effects in population dynamics

of the equilibrium solutions to these equations. That is, if $\frac{\partial^{2} f}{\partial I^{2}} \leq 0$ holds, then, depending on the value of the basic reproduction number, the solutions with positive initial conditions converge either to the endemic equilibrium state (when $R_{0}>1$ ), or to the infection-free equilibrium state (when $R_{0} \leq 1$ ). It is remarkable that this global stability does not require, in any way, a dependence of $f(S, I)$ on $S(t)$. Ultimately, there is a possibility that the individuals who shift to Safe behaviour are completely protected (e.g. they are vaccinated in response to the threat), which implies that $\beta_{S}=0$. A natural assumption is that the vaccination rate, $h(S, I)$, increases with increasing infections, (that is $\frac{\partial h}{\partial I} \geq 0$ ). In a recent paper [52] it is proved that if this dependence is at least linear (that is, if $\frac{\partial^{2} h}{\partial I^{2}} \geq 0$ holds), and the earlier condition $\frac{\partial^{2} f}{\partial I^{2}} \leq 0$ still holds, then equilibrium solutions to the equations are again asymptotically stable.

In this approach we could, for instance, assume that $A(t)$ is the amount of money invested into promoting safe behaviour, and then further assume that $P_{S}(t)$ is an explicit function of $A(t)$. Furthermore, one can assume that the amount of money invested is, in its turn, a function of the number of infected. In such case we eventually arrive at a system with nonlinear incidence rate which is similar to system (3.1) or (3.2) above.

Up to now we have disregarded memory and history. To incorporate these we have to assume that the shifting of behaviour depends not only on the current state of the system (the particular value of variables $S(t)$ and $I(t)$ in this case), but must also depend on the history of the system and, at least, on the direction of the phase motion. An illustration of such behaviour is one in which the value of $A(t)$ at which an individual person $\iota$ switches to Safe mode is significantly greater than the value at which the person switches back. This type of memory is well recognised as arising in different areas related to the analysis of human behaviour such as, for example, sociology, economics, neuroscience, and cognitive science. In a general context it often is characterized as a 'first impressions last' phenomenon: once a person's mind is made up one way or the other, it is more difficult to change it to the opposite than it is to convince the person in the first place. As a manifestation of this phenomenon in economics, we mention the business concept of goodwill: the idea that there can be a monetary value attached to the customers that a business presently has. The idea goes far beyond the fact that the customers buy goods from the firm, to the respect that the customers have for this particular business. If the first time a consumer becomes a customer of a particular firm and is satisfied with the goods or services provided by the firm, then he or she might well decide to bring his/her custom to this firm. Thus, this consumer has a "goodwill" towards this particular firm. However, when a consumer becomes dissatisfied with his present supplier, he takes his custom elsewhere. The level of satisfaction at which a consumer abandons his supplier is not the same as that at which he, in the first instance, became a customer of this particular supplier.

Another example is slander. A mere retraction of a slur against a group or a person is hardly a satisfactory resolution. Damage has been done, and people will remember the slur. It is like trying to unring a bell. Many people will have lost their good opinion of the person, and will not return their favour until a great deal of trust is again built up. It is obvious that some form of time-dependence is required to model such situations, since very few of us are blessed with perfect memory. Such circumstances can be modelled using what is known in hysteresis as an non-ideal relay, of which more later.

These examples of systems with memory came from economics and social sciences. The behaviour that these examples describe occurs in other areas of human life as well; such behaviour should be true, for instance, for the human response to danger. Our aim is to demonstrate how this type of behaviour can be modelled mathematically and how it can influence the dynamics of an epidemic.

\subsection{Adiabatic limit of relationships between Influence Function $A(t)$ and proportion $P_{S}(t)$}

\subsubsection{Existence of an adiabatic limit}

We now proceed to derive our model from first principles, which below we refer to as "Assumptions". We begin with a very general assumption. 
A. Pimenov, T.C. Kelly, A. Korobeinikov, M.J.A. O’Callaghan, A.V. Pokrovskii, D. Rachinskii Memory effects in population dynamics

Assumption 3.1. A restriction $A_{\tau}(\cdot)$ of the input $A(\cdot)$ to an interval $0 \leq t \leq \tau$ uniquely defines the corresponding restriction $P_{S \tau}(\cdot)$.

This is the causality assumption, which states that the future behaviour of the input has no effect on the dynamics of $P_{S}$. In mathematical terms, Assumption 3.1 implies the existence of an operator, $W$, that relates the input function $A_{\tau}(\cdot)$ to the output function $P_{S_{\tau}}(\cdot)$, that is

$$
P_{S \tau}(\cdot)=W A_{\tau}(\cdot)
$$

where operator $W$ satiffies the Volterra property, namely for any $0<\sigma<\tau$, function $P_{S \sigma}(\cdot)=W A_{\sigma}(\cdot)$ must coincide with the restriction of function $P_{S \tau}(\cdot)$ to the interval $0 \leq t \leq \sigma$.

Let us suppose that $A(t)$ evolves very slowly, and hence $P_{S}$ (almost) reaches an equilibrium before the value $A(t)$ changes significantly. In other words, if at a certain moment $\tau$ we "freeze" $A$ such that $A(t)=A(\tau)$ holds for all $t \geq \tau$, then $P_{S}(t) \approx P_{S}(\tau)$ holds for all $t \geq \tau$ as well. This property is called the adiabatic limit existence. In order to define the adiabatic limit formally, we have to examine the "slow-time" limit of the process. For a given function $A(t), 0 \leq t \leq \tau$, we consider a hypothetical "slow" function

$$
A^{\gamma}(t)=A(\gamma t), \quad 0 \leq t \leq \tau / \gamma,
$$

for small $\gamma \ll 1$. This function varies very "slowly" with respect to time, and hence $\left|\dot{A}^{\gamma}\right| \ll 1$. By Assumption 3.1, there exists a well-defined counterpart $P_{S}^{\gamma}(\cdot)=W A^{\gamma}(\cdot)$.

Assumption 3.2. There exists a function $y(t)$ such that

$$
\lim _{\gamma \rightarrow 0}\left|y(t / \gamma)-P_{S}^{\gamma}(t / \gamma)\right|=0 .
$$

Assumption 2 defines the operator $\widetilde{W}$, which relates the $y(t)=\widetilde{W} A$ to the function $A(t)$. The operator $\widetilde{W}$ is the adiabatic limit of operator $W$.

\subsubsection{Non-ideal relay as an adiabatic limit of individual memory}

Let us consider a susceptible individual $\iota$ in isolation. As we mentioned in Section 3.1, there is a good reason to suggest that the relationship between $A(t)$ and $P_{S}(t)$ has a strong memory component. On an individual level, the idea is that the level of $A(t)$ when $\iota$ switches to Safe mode is significantly greater than the level when $\iota$ switches back. In other words, for all values $A(t) \geq \alpha_{S}(\iota)$ the only equilibrium mode of behaviour which the individual $\iota$ exhibits is a Safe mode of behaviour, whereas for all values $\left.A(t) \leq \alpha_{R}(\iota)<\alpha_{S}(\iota)\right)$ the only equilibrium behaviour which $\iota$ exhibits is a Risky mode. For $\alpha_{R}(\iota)<A(t)<\alpha_{S}(\iota)$ the individual $\iota$ has two possible modes of equilibrium behaviours: either Safe, or Risky. Finally, we assume that $\iota$ is "lazy": he or she does not change the mode of behaviour as long as the input $A(t)$ varies within the boundaries

$$
\alpha_{R}(\iota)<A(t)<\alpha_{S}(\iota)
$$

We create a correspondence between the input $A(t)$ and a function $x(t)=x(t, \iota)$, such that $x(t)=1$ if at moment $t$ in time the individual $\iota$ exhibits Safe behaviour, and $x(t)=0$ otherwise. We denote by $R_{\iota}$ the adiabatic limit of the relationship between $A(t)$ and $x(t)$. To describe this adiabatic limit, we need one more concept, that of what is termed a non-ideal relay (with thresholds $\alpha_{R}$ and $\alpha_{S}$, where $\left.\alpha_{R}<\alpha_{S}\right)$ which is the simplest hysteretic transducer. This transducer plays an important role below, and we include here an informal definition. The transducer's output $x(t), t \geq t_{0}$, can take either of two values, 0 or 1 , at any moment; the relay is then said to be switched off or switched on, respectively. The typical dynamics of the output is illustrated by Figure 1(a). The variable output

$$
x(t)=\left(\mathcal{R}_{\alpha_{R}, \alpha_{S}}\left[t_{0}, r_{0}\right] u\right)(t), \quad t \geq t_{0},
$$


A. Pimenov, T.C. Kelly, A. Korobeinikov, M.J.A. O’Callaghan, A.V. Pokrovskii, D. Rachinskii Memory effects in population dynamics

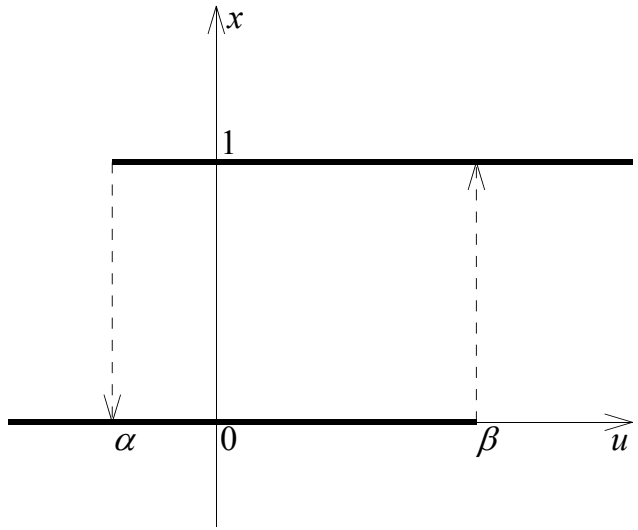

(a)

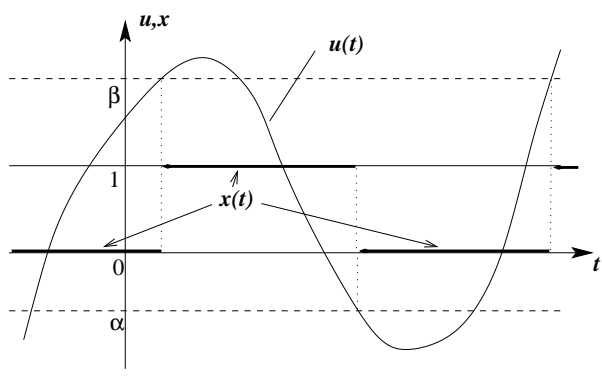

(b)

Figure 1. (a) Non-ideal relay, and (b) Input and output of the non-ideal relay (adapted from $[12])$.

depends on the continuous input variable $u(t), t \geq t_{0}$, and on the initial state $r_{0} \in\{0,1\}$. The output behaves 'lazily': it remains unchanged as long as the phase pair $(u(t), x(t))$ belongs to either of the two bold lines in Figure 1(a).

Figure 1(b) gives another geometrical illustration of the definition of the non-ideal relay. We combine on the same graph both an input and the corresponding output of the relay. From the physical point of view, the definition of the output is verifiable only for those inputs that do not have local minima equal to $\alpha_{R}$ and local maxima equal to $\alpha_{S}$. We arrive at the following assumption:

Assumption 3.3. In the adiabatic limit, the operator $R_{\iota}$ coincides with the non-ideal relay $\mathcal{R}_{\alpha_{R}, \alpha_{S}}$.

\subsubsection{Preisach nonlinearity as adiabatic limit of collective memory}

Assumption 3.4. We treat the total population as an infinite ensemble $\mathcal{J}$, and we assume that elements of $\mathcal{J}$ behave independently.

The reason for swapping a large finite set with an infinite set is purely technical: it is easier to integrate a continuous function, rather than to sum a finite series. As was explained in the previous section, to each individual element $\iota \in \mathcal{J}$ there correspond two numbers, $\alpha_{R}(\iota)$ and $\alpha_{S}(\iota)$, satisfying the inequalities $0 \leq \alpha_{R}(\iota)<\alpha_{S}(\iota) \leq 1$. The ensuing behaviours described above continue to hold.

Assumption 3.5. The pairs, $\left(\alpha_{R}(\iota), \alpha_{S}(\iota)\right), \iota \in \mathcal{J}$, of thresholds are distributed with some integrable density $\mu\left(\alpha_{R}, \alpha_{S}\right)$.

Assumption 3.6. The adiabatic limit of operator $W$ is described as

$$
\begin{aligned}
\left(\mathcal{P}\left[\eta_{0}\right] A\right)(t) & =\int_{0}^{1} \int_{\alpha_{R}}^{1} \eta\left(t, \alpha_{R}, \alpha_{S}\right) \mu\left(\alpha_{R}, \alpha_{S}\right) \mathrm{d} \alpha_{R} \mathrm{~d} \alpha_{S}, \\
\eta\left(t, \alpha_{R}, \alpha_{S}\right) & =\left(R_{\alpha_{R}, \alpha_{S}} A\right)(t) .
\end{aligned}
$$

This is an expression of the Preisach nonlinearity, which was originally introduced in the context of ferromagnetism, [68] and has since found more general applicability. In hydrology, for example, the independent domain model is equivalent to the Preisach nonlinearity, and was developed in parallel to that by Néel, [64], Everett \& Whitton, [29] and others. For a succinct explanation of the development of 
A. Pimenov, T.C. Kelly, A. Korobeinikov, M.J.A. O’Callaghan, A.V. Pokrovskii, D. Rachinskii Memory effects in population dynamics

the Preisach model and its relation to the independent domain model, see [62]. The function $\eta\left(t, \alpha_{R}, \alpha_{S}\right)$ is called the state of the Preisach nonlinearity, and $\eta_{0}\left(\alpha_{R}, \alpha_{S}\right)$ is the state at the initial moment of time. In what follows we use the notation $\mathcal{P}$ instead of $\mathcal{P}\left[\eta_{0}\right]$ if it does not lead to any ambiguity. The state $\eta\left(t, \alpha_{R}, \alpha_{S}\right)$ describes the distribution of population between the Risky and Safe modes of behaviour, and $\eta\left(t, \alpha_{R}, \alpha_{S}\right)$ is 0 whenever the corresponding part of the population chooses Risky behaviour, and 1 whenever it chooses Safe behaviour. It is a property of Preisach operator that the total memory of the continuum of the non-ideal relays $R_{\alpha_{R}, \alpha_{S}}$ at a moment $t_{1}$ is uniquely determined by the sequence of the previous minima and maxima $\left(t<t_{1}\right)$. According to the wiping-out property any maximum of $A(t)$ that is higher than some previous maxima or minimum that is lower than some previous minima erases these maxima and minima from the memory of the Preisach operator, so the recorded sequence of previous minima is always increasing and the sequence of previous maxima is always decreasing. This is called the shock type memory. For the detailed explanation of the evolution of the state $\eta\left(t, \alpha_{R}, \alpha_{S}\right)$ please refer to [62].

\subsection{Dynamics of $P_{S}(t)$ far away from an equilibrium}

\subsubsection{Equilibria of Influence Function}

Realistically, in the case of an epidemic $A(t)$ changes rather fast, and the idea of the adiabatic limit, as described in the previous subsection, may be an inadequate description of a real-life situation. In order to deal with the situations where $A(t)$ varies fast, in this subsection we apply the approach, which was developed in $[21,22]$ for modelling memory effects in multi-agents economics.

In this subsection we introduce an analogue of the matric potential mentioned above.

The Equilibrium Influence Function or Aggregated Stimuli is defined as being that hypothetical value $y$ of the input variable $A$ which, if instantaneously achieved by the actual input variable, would cause the population structure to remain constant. That is, if $A(t)=y$ for all $t \geq \tau$, then the activity level remains constant: that is $P_{S}(t)=P_{S}(\tau)$ for all $t \geq \tau$. It is not expected, however, that $A(t)$ will remain at the equilibrium level, and we have to emphasize that the behaviour in the areas separated from equilibrium is of interest. Furthermore, the equilibrium lavel $y(t)$ would likely depend on the prehistory of the problem. An obvious example of this would be that the equilibrium level, where $P_{S}$ remains constant, would be different depending on whether the current state were reached after a large epidemic or during a "quiet" period.

Assumption 3.7. At any given time moment $\tau$ there exists a unique equilibrium level, which is denoted $y=y(\tau)$.

The meaning of equilibrium rate $y(t)$ can be interpreted as the current state of the awareness of the population about the infection. If the external pressure or Influence Function $A(t)$ is equal to the current awareness $y(t)$, then the total number of people who choose the Safe mode of behaviour does not change; if $A(t)$ is greater than the current awareness $y(t)$, then more people will act more safely, and if $A(t)$ is less than $y(t)$, then people relax concerning the issue and $P_{S}(t)$ decreases. Thus, the following assumption holds:

Assumption 3.8. The number $P_{S}(t)$ changes smoothly, and its rate of change $\dot{P}_{S}(t)$ is proportional to the difference between the actual input variable $A(t)$ and the equilibrium level $y(t)$ :

$$
\dot{P}_{S}(t)=k(A(t)-y(t)) .
$$

The differential relationship (3.7) is not yet "closed", and hence it can not be solved numerically or analytically, nor can it produce results that could verify or falsify the assumptions made. In order to close the system, some relationship between the equilibrium level $y(t)$ and the current level of $P_{S}(t)$ is needed. 
A. Pimenov, T.C. Kelly, A. Korobeinikov, M.J.A. O’Callaghan, A.V. Pokrovskii, D. Rachinskii Memory effects in population dynamics

\subsubsection{Completing the model}

In order to complete the relationship between $P_{S}(\cdot)$ and $A(\cdot)$, the differential equation (3.7) must be closed by defining a relationship between $P_{S}(\cdot)$ and $y(\cdot)$. This relationship only admits certain pairs of functions $y(\cdot)$ and $P_{S}(\cdot)$. The totality of the pairs, $\left(P_{S}(\cdot), y(\cdot)\right)$, which are possible in our system, is denoted by $\Pi$, where both components, $P_{S}(\cdot)$ and $y(\cdot)$, are defined for the same interval $0 \leq t \leq \tau_{0}$. For a particular interval $[0, \tau]$, the subset of $\Pi$ consisting only of the pairs defined for $0 \leq t \leq \tau$ is denoted $\Pi_{\tau}$.

Assumption 3.9. The totality $\Pi$ is rate-independent; that is if a pair

$$
\left(P_{S}(\cdot), y(\cdot)\right), \quad 0 \leq t \leq \tau,
$$

is in $\Pi_{\tau}$, then for any positive $\gamma$ the pair $\left(P_{S}^{\gamma}(\cdot), y^{\gamma}(\cdot)\right)$, given by

$$
\left(P_{S}^{\gamma}(t), y^{\gamma}(t)\right)=\left(P_{S}(\gamma t), y(\gamma t)\right), \quad 0 \leq t \leq \tau / \gamma
$$

belongs to $\Pi_{\tau}^{\gamma}$.

This means that a scaling of the rate of change for the input results in the same scaling being applied to the output (see Figure 2). Note that a rate-independence for $\left(P_{S}(\cdot), A(\cdot)\right)$ or $(y(\cdot), A(\cdot))$ is hardly possible, as this means that transient processes are ignored. Biologically this assumption implies that there are individuals with a "long" memory in the population.

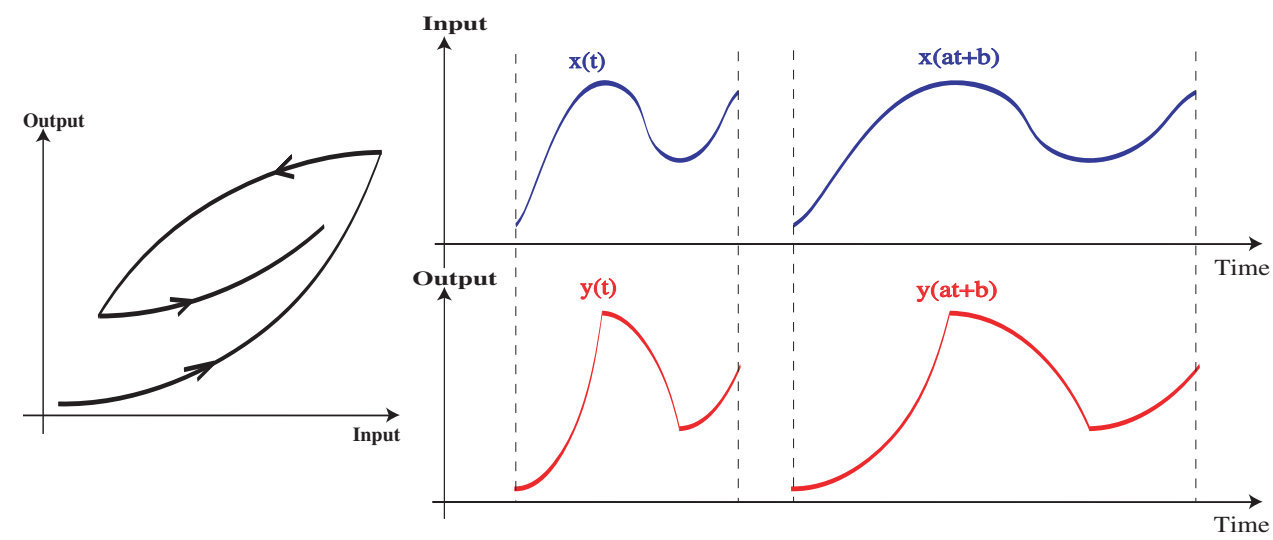

FIgURE 2. The action of a rate-independent operator on an input function $f(t)$ produces the output function $g(t)$. Rate-independence means that the action of the operator on the transformed input $f(\gamma(t))$, where $\gamma(t)$ is monotonically increasing, produces a similarly scaled output, $g(\gamma(t))$.

Assumption 3.10. For a given function $y(t), 0 \leq t \leq \tau$, there exists exactly one function $P_{S}(t), 0 \leq$ $t \leq \tau$, such that $\left(P_{S}(\cdot), y(\cdot)\right) \in \Pi$.

This is equivalent to the hypothesis that for any admissible function $y(t), 0 \leq t \leq \tau$, the corresponding value $P_{S}(\tau)$ is uniquely defined. However, we have to emphasize that the value $P_{S}(\tau)$ cannot be uniquely defined in terms of the value $y(\tau)$ only. Taking into account Assumption 3.9, it is clear that all relevant information about the prehistory $y_{\tau}(\cdot)$ may be condensed into the form of a sequence $S V\left(y_{\tau}(\cdot)\right)$ of the shock values, i.e. into the sequence of alternating locally maximal and locally minimal values of the 
A. Pimenov, T.C. Kelly, A. Korobeinikov, M.J.A. O’Callaghan, A.V. Pokrovskii, D. Rachinskii Memory effects in population dynamics

function $y_{\tau}(\cdot)$. In mathematical terms, this means that there must exist an operator $G$, which relates each unique function $P_{S}(\cdot)$ to its "companion" in $\Pi$, i.e. $P_{S}(\cdot)=G y(\cdot)$, such that for any $t \in[0, \tau]$ the value $y(t)$ is the equilibrium level for function $P_{S}(\cdot)$ at the instant $t$. From Assumption 3.9, it is clear that the operator $G$ must also be rate-independent. The general relationship (3.7) can now be written as

$$
\begin{aligned}
\dot{P}_{S}(t) & =k(A(t)-y(t)), \\
P_{S}(\cdot) & =G y(\cdot) .
\end{aligned}
$$

All that remains, in order to close the system, is to find a useful and justifiable form for the rateindependent operator $G$. The theory of rate-independent memory operators has been developing rapidly in recent years. Such operators are called hysteresis operators. The reader is referred again to the fundamental texts $[13,54,61,73]$ and to the recent three-volume set [70] which surveys the current state of research in hysteresis.

In order to justify a particular form for the operator $G$, the "slow-time" limit of the process is examined. Given a function $y(t), 0 \leq t \leq \tau$, and $P_{S}(\cdot)=G y(\cdot)$, consider a hypothetical "slow" function

$$
y^{\gamma}(t)=y(\gamma t), \quad 0 \leq t \leq \tau / \gamma,
$$

for small $\gamma \ll 1$. This function varies very "slowly" with respect to time, so $\left|\dot{y}^{\gamma}\right| \ll 1$. By Assumption 3.10 , there exists a well-defined counterpart

$$
P_{S}^{\gamma}=G y^{\gamma} .
$$

Moreover, by Assumption 3.9, this function satisfies

$$
P_{S}^{\gamma}(t)=P_{S}(\gamma t)
$$

In particular, there exists an input function $A^{\gamma}(t)$ such that

$$
\begin{aligned}
& \dot{x}^{\gamma}(t)=k\left(A^{\gamma}(t)-y^{\gamma}(t)\right), \\
& x^{\gamma}(\cdot)=G y^{\gamma}(\cdot) .
\end{aligned}
$$

Since everything is "slow", the derivative $\dot{x}^{\gamma}$ is very small, and thus for sufficiently small $\gamma$ we have

$$
A^{\gamma}(t) \approx y^{\gamma}(t) .
$$

From Assumption 3.1 it follows that the function $x^{\gamma}(\cdot)$ can be understood as

$$
x^{\gamma}(\cdot)=W A^{\gamma}(\cdot) \approx G A^{\gamma}(\cdot),
$$

since $A^{\gamma}(t) \approx y^{\gamma}(t)$. However we found this limit above in Subsection 3.2.3 (see Assumption 3.6). Therefore, the following assertion holds:

Proposition 3.11. Operator $G$ coincides with the operator (3.6).

Returning to statements (3.11) and (3.12), the relations become

$$
\begin{aligned}
\left(G y^{\gamma}\right)(t) & =\int_{0}^{1} \int_{\alpha_{R}}^{1} \eta\left(t, \alpha_{R}, \alpha_{S}\right) \mu\left(\alpha_{R}, \alpha_{S}\right) \mathrm{d} \alpha_{R} \mathrm{~d} \alpha_{S}, \\
\eta\left(t, \alpha_{R}, \alpha_{S}\right) & =\left(R_{\alpha_{R}, \alpha_{S}} y^{\gamma}\right)(t) .
\end{aligned}
$$

Thus, the principal system of equations can now be written as

$$
\begin{aligned}
\dot{x}(t) & =k(A(t)-y(t)), \\
x(t) & =\int_{0}^{1} \int_{\alpha_{R}}^{1} \eta\left(t, \alpha_{R}, \alpha_{S}\right) \mu\left(\alpha_{R}, \alpha_{S}\right) \mathrm{d} \alpha_{R} \mathrm{~d} \alpha_{S}, \\
\eta\left(t, \alpha_{R}, \alpha_{S}\right) & =\left(R_{\alpha_{R}, \alpha_{S}} y^{\gamma}\right)(t) .
\end{aligned}
$$


A. Pimenov, T.C. Kelly, A. Korobeinikov, M.J.A. O’Callaghan, A.V. Pokrovskii, D. Rachinskii Memory effects in population dynamics

Once a density function $\mu\left(\alpha_{R}, \alpha_{S}\right)$ (which is sometimes called a Preisach function) is specified, this is a closed operator-differential system of equations. It is an open question, what form of density is suitable for use in the epidemiological context. Below for demonstration purposes we consider the simplest case assuming uniform density. In another notation, system (3.14) can be expressed as

$$
\begin{aligned}
\dot{x}(t) & =k(A(t)-y(t)), \\
x(t) & =\left(\mathcal{P}\left[\eta_{0}\right] y\right)(t) .
\end{aligned}
$$

The key feature of this equation, which is more clearly seen in the compact notation, is that the action of the Preisach nonlinearity is under the highest derivative in the equations. This contrasts with the relatively well studied case of the Preisach nonlinearity on the right-hand side of such operatordifferential equations (i.e. $\dot{x}=f(x, t,(\mathcal{P} x)(t)))$. Some mathematical properties of solutions of system (3.15), such as the existence and the uniqueness of solution, have been addressed; see $[4,55,56]$ and the references therein. What remains in this application is to examine the behaviour of this system in a qualitative sense, and assess whether it could be applicable in an epidemiological context. The next section attempts to address this question.

\section{Finalising the Model and Numerical Experiments}

\subsection{Finalising the model}

Equations (2.5), (3.7) can be now finalised using equation (3.15). This yields

$$
\begin{aligned}
\dot{S} & =b-\left(\beta_{R}\left(1-P_{S}\right)+\beta_{S} P_{S}\right) S I-\mu S, \\
\dot{I} & =\left(\beta_{R}\left(1-P_{S}\right)+\beta_{S} P_{S}\right) S I-(\gamma+\mu+\sigma) I, \\
\dot{P}_{S}(t) & =k(A(t)-y(t)), \\
P_{S}(t) & =\left(\mathcal{P}\left[\eta_{0}\right] y\right)(t) .
\end{aligned}
$$

This set of equations provides a SIR model which has two susceptible groups, each with its own incidence rate, $\beta_{S}$ and $\beta_{R}$, with $\beta_{S}<\beta_{R}$. The sizes $P_{S}(t)$ and $P_{R}(t)$ of each group are given as fractions of the total susceptible population, with $P_{R}(t)=1-P_{S}(t)$, and the fraction $P_{S}(t)$ is determined by the Influence Function $A(t)$.

In this paper we model the relationship between $P_{S}(t)$ and $A(t)$ using a Preisach reservoir model, which provides two major features: $(i)$ a delay in time between a change in stimuli and the change in $P_{S}(t)$, and $(i i)$ a strong nonlinearity induced by Preisach memory. To obtain a better understanding of the impact of memory effects on the model dynamics, we also consider, for the purpose of comparison, the following kinds of relationships between $P_{S}$ and $A$ :

1. $P_{S}(t)=f(y(t))$ : there is a delay, but no memory;

2. $P_{S}$ is given by (4.1) with a density which is constant in some triangle (e.g. $0 \leq \alpha_{R} \leq \alpha_{S} \leq 1$ ), and is zero outside of this triangle.

Note that $P_{S}$ may be also defined by (4.1) with non-uniform density; however, this type of relationship is outside of the scope of this paper.

Choosing an appropriate function $f(A)$ for Type 1 relationship may be not a straightforward task. The simplest assumption about the structure of the Preisach operator is that the Preisach measure is equal to 1 in the triangle $0 \leq \alpha_{R} \leq \alpha_{S} \leq 1$, and to 0 outside of this triangle. If $y(t)$ is monotonically increasing from the value $y(0)=0$, then the output of such an operator is defined by the formula $P_{S}(t)=(\mathcal{P} y)(t)=(y(t))^{2}$. For the purpose of comparison it is adequate, therefore, to choose $f(y)=y^{2}$. 
A. Pimenov, T.C. Kelly, A. Korobeinikov, M.J.A. O’Callaghan, A.V. Pokrovskii, D. Rachinskii Memory effects in population dynamics

This function is frequently used in association with the learning curve of the predator in biological systems (for example, one of the possible interpretations of Holling type III functional response is that the predator learns how to handle the prey more effectively with the increase of the prey population size).

Here we will focus on the Influence Function $A(t)$ described by the formula

$$
A(t)=\kappa I(t)+A_{0}, \quad \kappa>0, \quad A_{0} \geq 0 .
$$

To study the impact of intervention and memory on the system dynamics, we introduce the effective basic reproduction number $\widetilde{R_{0}}$. We assume that the basic reproduction number $R_{0}$ is an intrinsic property of the system, whereas $\widetilde{R_{0}}$ reflects the impact of an intervention policy. For the model defined in equation set (4.1), the effective basic reproduction number can depend on the history, and it can be expressed as

$$
\widetilde{R_{0}}=\frac{b\left(\beta_{R}\left(1-P_{S}\left(Q_{0}\right)\right)+\beta_{S} P_{S}\left(Q_{0}\right)\right)}{\mu(\gamma+\mu+\sigma)},
$$

where $P_{S}\left(Q_{0}\right)$ is the value of $P_{S}$ at an infection free equilibrium state. It is not necessarily equal to zero, as emergence of a pathogen can be anticipated, and pro-active intervention measures can be taken prior to its actual appearance in a community.

\subsection{Method of numerical simulation}

Simulations can be evaluated using numerical techniques such as are presented by Flynn \& Rasskazov in [32]. For numerical experiments we take $b=\mu=0.1$ year $^{-1}, \beta=120$ year $^{-1}, \gamma=365 / 4=91.25$ year $^{-1}$ (e.g. infectious period of 4 days), $I(0)=0.001$ and $S(0)=0.5$ (c.f. with example in Section 2.1.1; we also remind that $I(t)$ and $S(t)$ are fractions). For these parameters $R_{0} \approx 1.31$, and hence the infection persists (see Fig. $3(\mathrm{a})$ ) in the population.

Suppose now that $P_{S}(t)=f(y(t))$; that is we replace the Preisach operator $\mathcal{P}$ in (4.1) with a functional operator $G y=f(y(t))$. Formula (4.2) implies that $P_{S}\left(Q_{0}\right)=f\left(A_{0}\right)$ at an infection-free equilibrium state, and hence the effective basic reproduction number is

$$
\widetilde{R_{0}}=\frac{b\left(\beta_{R}\left(1-f\left(A_{0}\right)\right)+\beta_{S} f\left(A_{0}\right)\right)}{\mu(\gamma+\mu+\sigma)} .
$$

This SIR model, having two susceptible groups with different incidence rates, has been very well studied [41]. For $\widetilde{R_{0}}<1$ the infection-free equilibrium of system (4.1) is globally asymptotically stable, and the endemic equilibrium is unstable, whereas for $\widetilde{R_{0}}>1$ the endemic equilibrium is globally asymptotically stable while the infection-free equilibrium is unstable. (The same is also true for the adiabatic limit of this model, where $P_{S}(t)=f(A(t))$.) At the endemic equilibrium state $\left(I^{*}, S^{*}, P_{S}^{*}\right)$, the following equalities hold:

$$
\begin{aligned}
P_{S}^{*} & =f\left(\kappa I^{*}+A_{0}\right), \\
S^{*} & =b-\frac{\gamma+\mu+\sigma}{\mu} I^{*}, \\
0 & =\left(\beta_{R}\left(1-P_{S}^{*}\right)+\beta_{S} P_{S}^{*}\right) S^{*} I^{*}-(\gamma+\mu+\sigma) I^{*} .
\end{aligned}
$$

For $f(y)=y^{2}, \beta_{R}=120$ year $^{-1}, \beta_{S}=1$ year $^{-1}, A_{0}=0$, and the other parameters as above, we obtain $P_{S}^{*} \approx 0.0482$ (see Fig. $3(\mathrm{~b})$ ).

\subsection{Model with the Preisach operator}

To demonstrate the effects of the Preisach operator, we choose $A_{0}=0, \kappa=1000, k=1$ year $^{-1}$, $S(0)=0.999$ and $I(0)=0.00001$, and compare the dynamics of model defined by equation set (4.1) with $P_{S}(t)=f(y(t))=y^{2}(t)$, which was described in the previous subsection, to this with $P_{S}(t)=\mathcal{P} y(t)$. 
A. Pimenov, T.C. Kelly, A. Korobeinikov, M.J.A. O'Callaghan, A.V. Pokrovskii, D. Rachinskii Memory effects in population dynamics

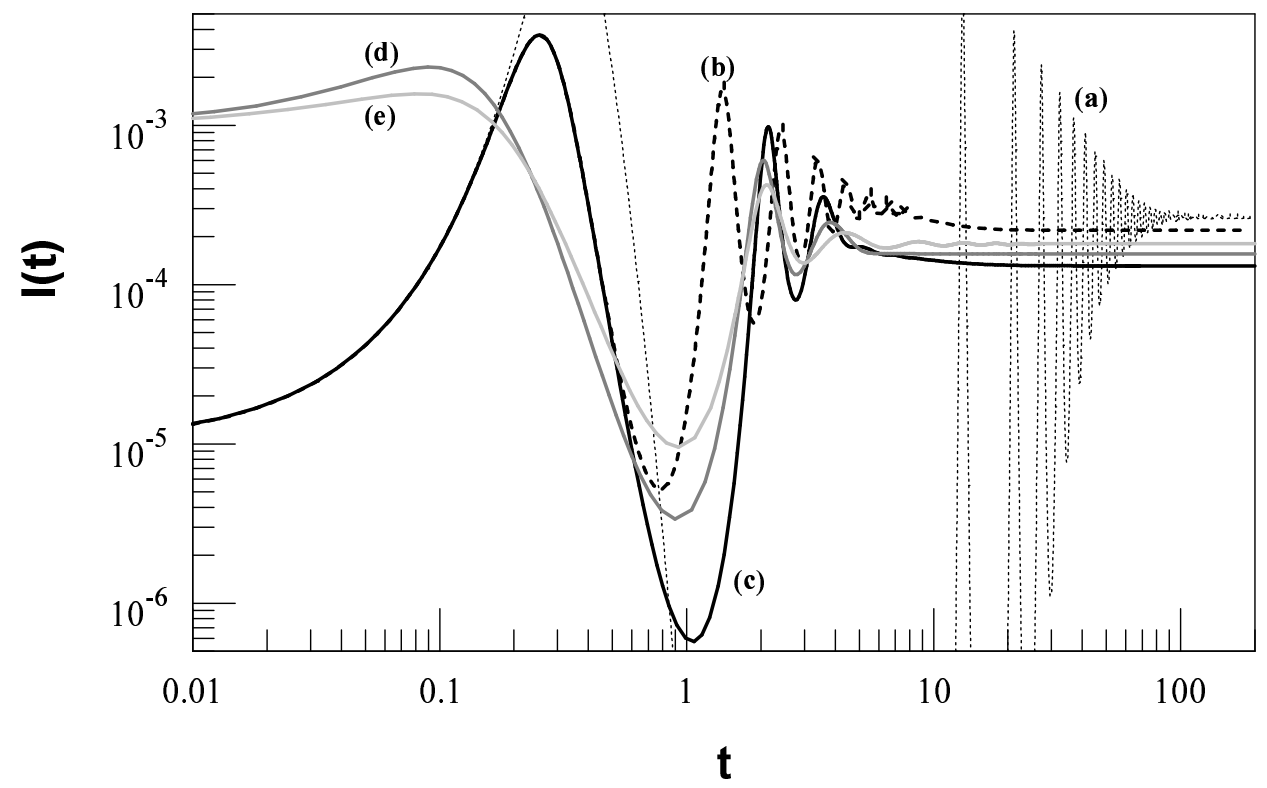

Figure 3. Dynamics of the infected sub-populations for the following models: (a) (dotted line) model (2.5); (b) (dashed line) non-hysteretic model (4.1) with $P_{S}(t)=f(y(t))$; (c)-(e) (solid lines) hysteretic model (4.1). Here the parameters are: $b=\mu=0.1$ year $^{-1}$, $\beta=120$ year $^{-1}, \gamma=365 / 4=91.25$ year $^{-1}, y(0)=0.01, \kappa=1000, k=1$ year $^{-1}$. Initial conditions are: $I(0)=10^{-5}$ and $S(0)=0.999$ for $(\mathrm{a})-(\mathrm{c}), I(0)=0.001, S(0)=0.9$ for $(\mathrm{d}) ; S(0)=0.85$ for $(\mathrm{e})$. Note that for the hysteric model there is a continuum of equilibrium states; the solutions (c)-(e) of the hysteretic model converge to different equilibrium state of this continuum, depending on initial conditions.

Curves (c)-(e) in Figure 3, which correspond to the latter case, suggest that for this model the hysteresis induces phenomena analogous to physical friction with respect to the spread of infection. That is, there are losses in the number of infected due to hysteresis, and the larger is the maximum value of $I(t)$ (and hence of $A(t)$ ), the lower is the equilibrium value $I^{*}$. Similar phenomena were observed in other systems with hysteresis, for example in ferromagnetic hysteresis. Another fundamental hysteretic effect, which is clearly seen on Figure 3, is that the trajectories of the model with hysteresis do not converge to a unique equilibrium state. To demonstrate this phenomenon, we vary the initial values $S(0), I(0)$. It is noteworthy that when the initial point $\left(S(0), I(0), P_{S}(0)\right)$ coincides with the endemic equilibrium point of the non-hysteretic model, then the endemic equilibrium states of the hysteretic and non-hysteretic models coincide. However, a variation of the initial conditions shifts the equilibrium point towards the lesser value of $I^{*}$, as seen in Figure 3.

We assume now that $A_{0}>0$, choosing $A_{0}=0.4$. For the nonzero $A_{0}$, the solutions, which are initiated near the endemic equilibrium state of the non-hysteretic model, converge to an endemic equilibrium state, which is located near to that of the non-hysteretic model (see curves (a,b,c,e) in Figure 4). (Note that curves (e) reappear after a prolonged time.) However, if the initial conditions are located further from the endemic equilibrium state of the non-hysteretic model, then the hysteresis can lead to the disappearance of infection. Curve (d) in Figure 4 shows such behaviour: here the infection disappears after a single outbreak of the disease. 
A. Pimenov, T.C. Kelly, A. Korobeinikov, M.J.A. O’Callaghan, A.V. Pokrovskii, D. Rachinskii Memory effects in population dynamics

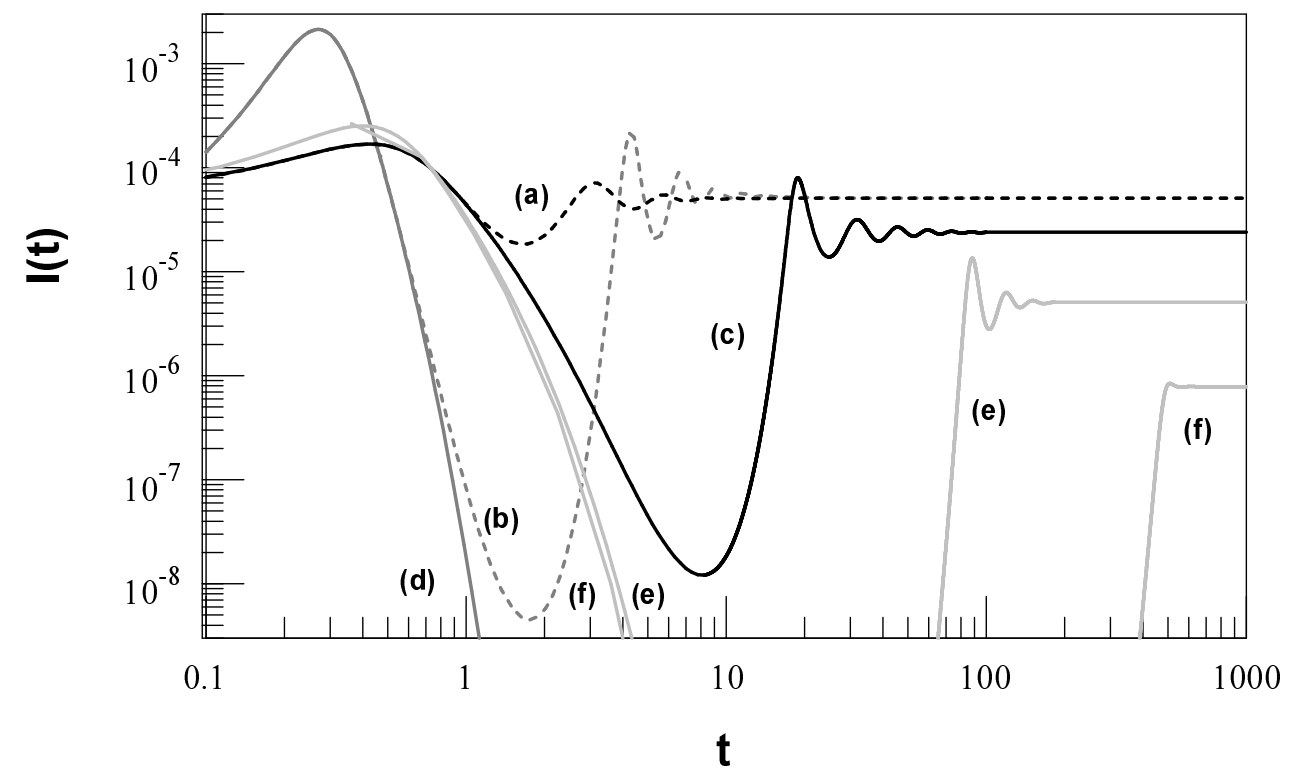

FiguRE 4. The level of infection $I(t)$ for non-hysteretic and hysteretic models. Here, curves (a) and (b) (dashed) are for $P_{S}(t)=y^{2}(t)$; curves (c),(d),(e) and (f) (solid) for $P_{S}(t)=\mathcal{P} y(t)$ with $A_{0}=0.4$. The parameters are $y(0)=0.01, S(0)=0.999$ and $I(0)=0.00001$ for $(\mathrm{a})$ and $(\mathrm{c})$, and $y(0)=0.4, S(0)=0.9535$ and $I(0)=5.09 \times 10^{-5}$ for (b) and (d). Parameters for (e) and (f) are the same as for (d) apart from $S(0)$, which is equal to 0.97 for (e) and to 0.973 for (f). Note that, depending on initial conditions, the solutions (c)-(f) of the hysteretic model converge to different equilibrium state of the continuum; c.f. Fig. 3

\subsection{Equilibrium sets and global dynamics of the model}

We have mentioned that the long term dynamics of the classical SIR model (2.5) without memory is determined by the value of the parameter $R_{0}$. The infection-free equilibrium of this model always exists and is globally stable for $R_{0}<1$. For $R_{0}>1$, the endemic equilibrium is globally stable (in the invariant domain $I>0, S \geq 0$ ), while the infection-free equilibrium becomes unstable (a saddle point). At the critical value $R_{0}=1$, the endemic and infection-free equilibria collide in a transcritical bifurcation and they exchange stability. As we mentioned in subsection 4.2, the same is true for the Risky-Safe SIR model without hysteresis, with the only difference being that parameter $\widetilde{R_{0}}$ is the bifurcation parameter in this case. The numerical simulations for model defined by the equations (4.1), (4.2) show that the trajectories of this system converge to an equilibrium state as well (see Figure 3). However, in contrast to simple model expressed in (2.5), this more complicated system has multiple coexisting equilibria which form a continual set with one or two connected components (represented by segments of curves in the phase space). One connected component of the equilibrium set, or its connected part, is a global attractor in the invariant domain $I>0, S \geq 0$. Moreover, each trajectory, after a transient dynamics, stabilises at an equilibrium point of the attractor; this particular equilibrium point depends on the initial conditions.

Effectively, the introduction of memory into the system in (2.5), thereby transforming it into system in equations (4.1), (4.2), results in the transformations of the isolated infection-free equilibrium into a connected continuum of infection-free equilibria, and of the isolated endemic equilibrium into a connected continuum of endemic equilibria. These two continua can merge into one connected set as the parameters are varied. The reason for multiple equilibria is that system (4.1), (4.2) has an internal variable, namely the proportion $P_{S}(t)$ of the population that has adapted Safe mode of behaviour. This additional degree 
A. Pimenov, T.C. Kelly, A. Korobeinikov, M.J.A. O’Callaghan, A.V. Pokrovskii, D. Rachinskii Memory effects in population dynamics

of freedom accounts for the coexistence of equilibrium states which have different values of this proportion that range over a certain interval. Another effect of the shock type memory (see section 3.2.3) is that the target equilibrium for a particular trajectory is determined by the shock value of variable $y$, which follows the shock value of the infected population $I$. In particular, the maximal shock value determines whether a trajectory stabilises at an endemic or an infection free equilibrium state. We now discuss these specific features of model (4.1), (4.2) in more detail.

At an equilibrium state, populations $I$ and $S$, as well as variable $y$ and variable $P_{S}$, measuring the fraction of population that adapted Safe behaviour, are constant. Moreover, the state $\eta\left(t, \alpha_{R}, \alpha_{S}\right)$ of the Preisach operator, which describes the distribution of population between the Risky and Safe modes of behaviour (see (3.6)), is constant in time as well. It is convenient to use the variable

$$
r(t)=\frac{b\left(\beta_{S} P_{S}(t)+\beta_{R}\left(1-P_{S}(t)\right)\right)}{\mu(\gamma+\mu+\sigma)}
$$

instead of variable $P_{S}(t)$, when discussing equilibria of system (4.1), (4.2). Accordingly, we will describe an equilibrium state by the quadruplet $\left(I^{*}, S^{*}, y^{*}, r^{*}\right)$. If it is an infection-free equilibrium, then, by (4.3), $r^{*}=\widetilde{R_{0}}$. It should be noted that, typically, one quadruplet $\left(I^{*}, S^{*}, y^{*}, r^{*}\right)$ represents infinitely many equilibrium states characterised by the same value of $r=r^{*}$ but different states $\eta\left(t, \alpha_{R}, \alpha_{S}\right)$ of the Preisach operator. (This reflects different distributions of the population between the two modes of behaviour with the same 'mean' $P_{S}$ and hence the same $r$ ). In general, a state of the Preisach operator is important, as nonstationary trajectories starting at the same values of $I, S, y$ and $r$, as a rule, have different components $I$ and $S$ for $t>0$. However, our numerical results give a strong indication that the stability of an equilibrium state of system (4.1), (4.2) is defined by the sign of the number $r^{*}-1$, as it is the case of system (2.5) without memory. Hence, the state of the Preisach operator is not essential for the following discussion of the equilibrium set and the attractor of system (4.1), (4.2).

Equilibrium states $\left(I^{*}, S^{*}, y^{*}, r^{*}\right)$ can be found by equating the right-hand sides of the first three equations of system (4.1) to zero. This yields

$$
b-\beta(r) S I-\mu S=0, \quad \beta(r) S I-(\gamma+\mu+\sigma) I=0, \quad y=\kappa I+A_{0},
$$

where $\beta(r)=r \mu(\gamma+\mu+\sigma) / b$. These equations should be combined with the inequalities

$$
y^{2} \leq P_{S} \leq 2 y-y^{2}
$$

for

$$
P_{S}=\frac{1}{\beta_{R}-\beta_{S}}\left(\beta_{R}-\frac{r \mu(\gamma+\mu+\sigma)}{b}\right),
$$

which follow from the last equation in (4.1). System (4.5), (4.6) has a set of solutions where $I=0$, $S=b / \mu$, and $y=A_{0}$, with $r$ ranging over the interval

$$
\frac{b\left(\left(\beta_{S}-\beta_{R}\right)\left(2 A_{0}-A_{0}^{2}\right)+\beta_{R}\right)}{\mu(\gamma+\mu+\sigma)} \leq r \leq \frac{b\left(\left(\beta_{S}-\beta_{R}\right) A_{0}^{2}+\beta_{R}\right)}{\mu(\gamma+\mu+\sigma)} .
$$

These solutions are infection free equilibria of system (4.1), (4.2). They form a segment $E_{0}$ in the hyperplane $I=0$ of the four dimensional space $(I, S, y, r)$. Dividing the second equation of system (4.5) by $I$ and then using the two other equations of this system and inequalities (4.6), we obtain the equalities

$$
I=\frac{b(r-1)}{r(\gamma+\mu+\sigma)}, \quad S=\frac{b}{r \mu}, \quad y=A_{0}+\frac{\kappa b(r-1)}{r(\gamma+\mu+\sigma)}, \quad y^{2} \leq P_{S} \leq 2 y-y^{2},
$$

which define endemic equilibria of system (4.1), (4.2). These equilibria form a curve segment $E_{\text {end }}$ in the space $(I, S, y, r)$. 
A. Pimenov, T.C. Kelly, A. Korobeinikov, M.J.A. O’Callaghan, A.V. Pokrovskii, D. Rachinskii Memory effects in population dynamics

Figure 5 shows how the projection $I_{\min }^{*} \leq I \leq I_{\max }^{*}$ of the curve $E_{\text {end }}$ of the endemic equilibria on $I$-axis depends on parameter $A_{0}$. The maximum and minimum endemic equilibrium values of $I$, that is, curves $I_{\max }^{*}=I_{\max }^{*}\left(A_{0}\right)$ and $I_{\min }^{*}=I_{\min }^{*}\left(A_{0}\right)$ in Figure 5 , have been found from the cubic equations, which correspond to the equalities in the inequality $y^{2} \leq P_{S} \leq 2 y-y^{2}$. These curves cross line $I=0$ at the values

$$
\begin{gathered}
A_{0}=A_{\max }^{*}=\sqrt{\frac{b \beta_{R}-\mu(\gamma+\mu+\sigma)}{b\left(\beta_{R}-\beta_{S}\right)},} \\
A_{0}=A_{\min }^{*}=1-\sqrt{1-\left(A_{\max }^{*}\right)^{2}},
\end{gathered}
$$

respectively. Hence, for $0 \leq A_{0}<A_{m i n}^{*}$, the curve $E_{\text {end }}$ of the endemic equilibria is located in the domain $I>0$ and does not intersect with the segment $E_{0}$ of the infection free equilibria, which is located in the hyperplane $I=0$ (see Figure 6a). As $A_{0}$ increases towards value $A_{m i n}^{*}$, the curve segment $E_{\text {end }}$ moves down towards hyperplane $I=0$ in the $(I, S, y, r)$ space ( $I$ is assumed to be a vertical direction), while the segment $E_{0}$ moves towards smaller values of $r$ along hyperplane $I=0$, and these two segments approach each other. At the bifurcation point $A_{0}=A_{\text {min }}^{*}$, the lower end of the curve segment $E_{\text {end }}$ merges with one end of the segment $E_{0}$. For $A_{\text {min }}^{*}<A_{0}<A_{\text {max }}^{*}$, two segments intersect, forming a "cross" shape (Figure 6c), that is, the equilibrium set becomes connected. We note that the lower bar of this cross lies in the domain $I<0$ and is therefore biologically irrelevant. As $A_{0}$ crosses the second bifurcation value $A_{\text {max }}^{*}$, the segments $E_{\text {end }}$ and $E_{0}$ disconnect again, as the segment $E_{\text {end }}$ moves to the domain $I<0$ completely (Figure 6e). Hence, for $A_{0}>A_{\max }^{*}$, all the nonnegative equilibria of system (4.1), (4.2) are infection free.

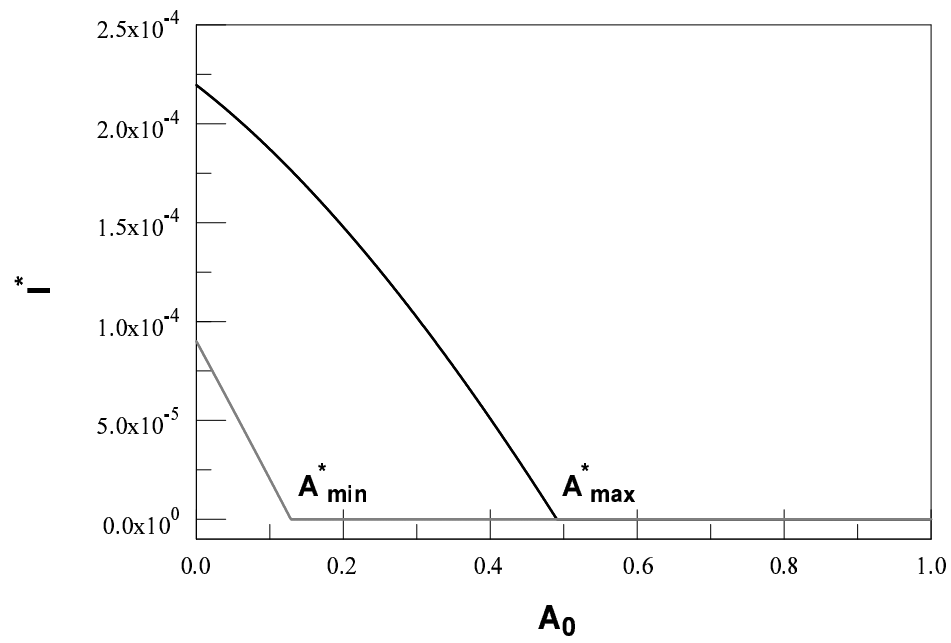

FiguRE 5. Projection of the curve $E_{\text {end }}$ of the endemic equilibrium states on $I$-axis depending on parameter $A_{0}$. The $I$-component of the endemic equilibria ranges between the curves $I_{\text {max }}^{*}=I_{\text {max }}^{*}\left(A_{0}\right)$ and $I_{\text {min }}^{*}=I_{\text {min }}^{*}\left(A_{0}\right)$.

A rigourous local stability analysis can be performed using an adaptation of the technique developed in $[4,5,11,22,55,66,67]$ for stability analysis of equation (3.15). In particular, using this technique it is possible to show that for all $r^{*}<1$ in a neighbourhood of the infection free equilibrium set the $y$-component of a solution must decrease. In this case system (4.1), (4.2) becomes locally equivalent to an ordinary differential equation system with the same equilibrium. It can be also expected that a more careful argument about monotonicity of the $y$-component of a solution could be combined with 
A. Pimenov, T.C. Kelly, A. Korobeinikov, M.J.A. O’Callaghan, A.V. Pokrovskii, D. Rachinskii Memory effects in population dynamics
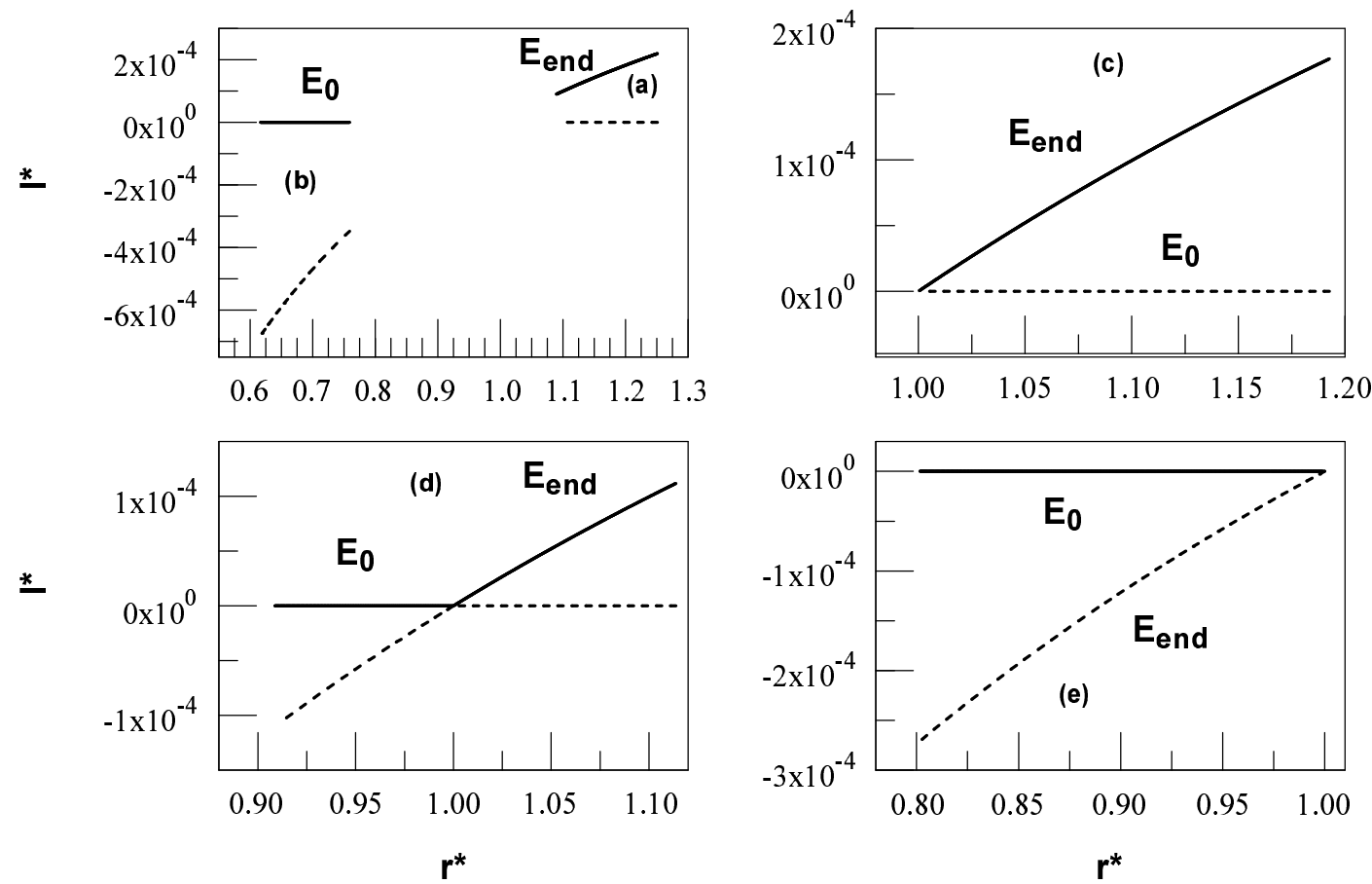

Figure 6. Projection of the equilibrium sets $E_{0}$ and $E_{\text {end }}$ on the $(r, I)$-plane for different values of parameter $A_{0}$. Segments of the stable equilibria are shown by the bold lines, the unstable equilibria is shown by the dashed lines. (a) Case $0 \leq A_{0}<A_{\min }^{*}$. There are two disjoint segments, and the positive set $E_{\text {end }}$ is the global attractor, while $E_{0}$ is unstable. (b) Case $A_{0}>A_{\max }^{*}$. The equilibrium set is the union of two discoint sets, where $E_{\text {end }}$ is below the abscissa; $E_{0}$ is the global attractor. (c) Case $A_{0}=A_{\min }^{*}$. The sets $E_{\text {end }}$ and $E_{0}$ connect forming a "corner" shape. (d) Case $A_{\min }^{*}<A_{0}<A_{\max }^{*}$. The cross shape is formed. The positive part of $E_{\text {end }}$ and the left bar of $E_{0}$ form the global attractor, whereas the right bar of the cross is composed of the unstable equilibria. (e) Case $A_{0}=A_{\max }^{*}$. The equilibrium set is of "corner" shape, where the unstable set $E_{\text {end }}$ is below the abscissa $I=0$; the infection free equilibrium set $E_{0}$ is the global attractor.

an adaptation of the Lyapunov function method. Potentially, this can provide a tool for proving the global stability of the equilibrium set for arbitrary initial conditions. However, such a rigourous analysis of the stability is beyond the scope of this paper. The following observations are based on numerical solution, performed for a limited number of initial conditions, and will be supported by a discussion of their epidemiological interpretation.

Numerical simulations confirm that an infection-free equilibrium $\left(b / \mu, 0, A_{0}, r^{*}\right)$ is stable if $r^{*}<1$, and unstable if $r^{*}>1$, while all of the endemic equilibrium points with $I^{*}>0$ are stable. This behaviour is similar to the case of system (2.5) without memory. As each equilibrium point of system (4.1), (4.2) is an element of a continuous segment of equilibrium states, a stable equilibrium can be only neutrally stable. Note that $\operatorname{sign} I^{*}=\operatorname{sign}\left(r^{*}-1\right)$ for the equilibrium points of the set $E_{\text {end }}$, and hence $r^{*}>1$ holds for the positive components of the set $E_{\text {end }}$. Furthermore, simulations show that the curve $E_{\text {end }}$ of endemic equilibria is a global attractor in the domain $I>0, S \geq 0$ for all $A_{0}<A_{\text {min }}^{*}$. Figure 3 shows the convergence of trajectories from different initial points to different points of $E_{\text {end }}$ for $A_{0}=0$. The infection-free equilibria set has $r^{*}<1$ and is unstable for $A_{0}<A_{\min }^{*}$. For $A_{\min }^{*}<A_{0}<A_{\max }^{*}$, when the equilibrium sets $E_{0}$ and $E_{\text {end }}$ cross as in Figure $6 \mathrm{c}$, the value of $r^{*}$ at their intersection point $E_{0} \cap E_{\text {end }}$ is 
A. Pimenov, T.C. Kelly, A. Korobeinikov, M.J.A. O’Callaghan, A.V. Pokrovskii, D. Rachinskii Memory effects in population dynamics

$r^{*}=1$. This intersection point divides set $E_{0}$ of the infection-free equilibria into an interval of unstable equilibria (the right horizontal bar of the cross) where $r^{*}>1$, and an interval of stable equilibria (the left bar of the cross) where $r^{*}<1$. In this particular case, the connected set of the stable equilibria, which includes all positive points of set $E_{\text {end }}$, and all of the infection-free equilibrium points where $r^{*} \leq 1$ (i.e., the stable set is the union of the upper and the left bars of the cross), is the global attractor of system (4.1), (4.2) in the domain $I>0, S \geq 0$. Finally, for all $A>A_{\text {max }}^{*}$ there are no positive endemic equilibria, and the set $E_{0}$ of infection-free equilibria becomes the global attractor of the system; $r^{*}<1$ holds for all points of $E_{0}$.

The most complicated and interesting case is when $A_{\min }^{*}<A_{0}<A_{\max }^{*}$ and the global attractor is a connected set of equilibrium points which includes both the infection-free and the endemic equilibrium points, as in Figure 6c. Figure 4 shows the trajectories from different initial conditions converging to endemic and infection-free equilibria points. It appears that only those trajectories that correspond to a sufficiently large outbreak of the infection converge to points belonging to the infection-free equilibrium set. More precisely, if the $y$-component of a trajectory exceeds, at any moment in time, the value $Y$ defined by the relation

$$
Y=\frac{1}{2 A_{0}}\left(A_{0}^{2}+\frac{\beta_{R}}{\beta_{R}-\beta_{S}}-\frac{\mu(\gamma+\mu+\beta)}{b\left(\beta_{R}-\beta_{S}\right)}\right),
$$

then this trajectory converges to an infection-free equilibrium state. Otherwise it converges to an endemic equilibrium. The reason is the permanent memory in the system. The inequality $y(\tau)>Y$ implies that all individuals with $\alpha_{S} \leq Y$ adopted Safe behaviour by the moment $\tau$. Moreover, a fraction of these individuals, namely those with $\alpha_{R}<A_{0}$, maintain Safe behaviour permanently (for all $t>\tau$ ), and never switch back to Risky behaviour even if the infection completely disappears. The inequality $y(\tau)>Y$ ensures that this fraction is sufficiently large to guarantee the convergence of the trajectory to an infectionfree equilibrium state. Furthermore, according to the third equation of system (4.1), the dynamics of the $y$-component of the solution follows the dynamics of its $I$-component (with some delay). In particular, $y$ never exceeds $I$ if initially $y(0)<I(0)$. Hence, the infected population must exceed the critical value $Y$ to guarantee the convergence to an infection free equilibrium state.

An important question is what is the meaning of $\widetilde{R_{0}}$ for this system. As we mentioned before, at an infection-free equilibrium $\widetilde{R_{0}}=r^{*}$. Since $r^{*}<1$ implies stability of the infection-free equilibrium, $\widetilde{R_{0}}<1$ implies its stability as well. However if we obtain $\widetilde{R_{0}}>1$, and the system is not yet at equilibrium (e.g., it is at the initial moment of time), then the outcome also depends on the dynamics of $y(t)$. On the other hand, if the system converges to an endemic equilibrium, then $r^{*}>1$ and hence $\widetilde{R_{0}}>1$.

\section{Discussion}

Here we have introduced what we consider to be an important development in methodology by including hysteresis for the description of memory in models of the spread of infectious pathogens. A consequence of hysteresis effects is the appearance of a continuum of equilibrium states, instead of isolated equilibrium states of traditional models. In this continuum of equilibrium states, the actual equilibrium reached by the system being dependent on the prehistory dynamics. We have shown that in an epidemic situation potential victims can alter their behaviour from a Risky to a Safe mode. This change in behaviour produced a hysteresis effect in the course of the epidemic and, in particular, in subsequent epidemics. These findings are of considerable interest to gaining an understanding of epidemics among animal populations (epizootic) where "advertising campaigns" are not applicable. However, recent research suggests that a system of Public Information exists within animal society $[19,23,69,72,74,75]$. Heretofore there has not been clear- cut evidence that "information" from the infected-infectious compartment exists, or if it does exist, that it influences the behaviour of the susceptibles, and that is specifically promotes an alteration in their behaviour from Risky to Safe.

Public Information is considered to be based on the transmission and reception of stimuli. Therefore signals could be visual, aural, or olfactory [8]. For example, in studies of mate selection in doves (Columba 
A. Pimenov, T.C. Kelly, A. Korobeinikov, M.J.A. O’Callaghan, A.V. Pokrovskii, D. Rachinskii Memory effects in population dynamics

livia var. domestica) males that are obviously heavily infected with feather lice are rejected by females [16]. Indeed, in the general theories concerning the origin of sex and sexual selection, it is argued that infected males are rejected due to the visual patterns of their plumage [36]. Therefore visual information leads to a "safe" response by the female.

What is now of great interest is the possibility that other more subtle signals, particularly of an olfactory nature [1] may influence behaviour of susceptibles when in the company of infected-infectious individuals. This suggests a fruitful area for future lines of investigation, particularly in relation to novel pathogens $[15,42]$, both micro- and macro-parasites.

Acknowledgements. We mourn the passing of our esteemed and irreplaceable colleague Professor Alexei Pokrovskii, Resquiat in Pacem.

A.P. acknowledges support of SFB Project 787 of the DFG. A.K. was supported by the Mathematics Applications Consortium for Science and Industry, funded by the Science Foundation Ireland Mathematics Initiative Grant 06/MI/005. T.K., M.J.O'C. and A.P. were partially supported by the National Academy for Integration of Research and Teaching and Learning (NAIRTL) Grant "Development and implementation of two project based courses "Mathematical Modelling for Biologists". A.V.P. and D.R. were partially supported by the Russian Foundation for Basic Researches Grant 10-01-93112 and Federal Programme 'Scientists of Innovative Russia' Grant 2009-1.5-507-007.

\section{References}

[1] L. Amo, I. Galván, G. Tomás, J.J. Sanz, Predator odour recognition and avoidance in a songbird. Functional Ecology, 22 (2008), 289-293. doi:10.1111/j.1365-2435.2007.01361.x

[2] R.M. Anderson, R.M. May, Infectious Diseases of Humans: Dynamics and Control. Oxford University Press, Oxford, 1992.

[3] R. Antia, V.V. Ganusov, R. Ahmed, The role of models in understanding CD8+ T - Cell memory, Nature Reviews Immunology, 5 (2005), 101-111.

[4] B. Appelbe, D. Flynn, H. McNamara, J.P. O'Kane, A. Pimenov, A. Pokrovskii, D. Rachinskii, A. Zhezherun, Rateindependent hysteresis in terrestrial hydrology, A Vegetated Soil Model with Preisach Hysteresis. IEEE Control Systems Magazine, 1 (2009), 44-69.

[5] B. Appelbe, D. Rachinskii, A. Zhezherun, Hopf bifurcation in a van der Pol type oscillator with magnetic hysteresis. Physica B, 403 (2008), No. 2-3, 301-304.

[6] N.T. Bailey, The Mathematical Theory of Infectious Diseases (2-nd edition). Charles Griffin and Co. Ltd., 1975.

[7] P.B. Banks, C.R. Dickman, Alien predation and the effects of multiple levels of prey naivete. Trends Ecol. Evol., 22 (2007), No. 5, 229-230.

[8] C. Barnard, Animal Behaviour - Mechanism, Development, Function and Evolution. Pearson/ Prentice Hall, London, 2004.

[9] J.J. Bolhuis, C.D.L. Wynne, Can evolution explain how minds work?. Nature, 458 (2009), 832-833.

[10] J.J. Bolhuis, E.M. Macphail, A critique of the neuroecology of learning and memory. Trends Cogn. Sci., 5 (2001), 426-433.

[11] M. Brokate, A. Pokrovskii, D. Rachinskii, Asymptotic stability of continuum sets of periodic solutions to systems with hysteresis. J. Math. Anal. Appl., 319 (2006), No. 1, 94-109.

[12] M. Brokate, A. Pokrovskii, D. Rachinskii, O. Rasskazov, Differential equations with hysteresis via a canonical example. in The Science of Hysteresis (Bertotti and Mayergoyz, editors). Vol. 1, pp. 125-291, Elsevier Science, 2005. ISBN: 9780-12-480874-4

[13] M. Brokate, J. Sprekels, Hysteresis and Phase Transitions. Springer, New York, 1996.

[14] V. Capasso, Mathematical Structures of Epidemic Systems. Lecture Notes in Biomathematics, Vol. 97. Springer-Verlag, Heidelberg, 1993.

[15] C.F. Clancy, M.J.A. O'Callaghan, T.C. Kelly, A multi-scale problem arising in a model of avian flu virus in a seabird colony. J. Phys. Conf. Ser., 55 (2006), 45-54.

[16] D. Clayton, The influence of parasites on host-sexual selection, Parasitology Today, 7 (1991), 329-334

[17] N.S. Clayton, D.P. Griffiths, N.J. Emery, A. Dickinson, Elements of episodic memory in animals, Phil. Trans. R. Soc. Lond B, 356 (2001), 1483-1491.

[18] T.S. Collett, M. Collett, Memory use in insect visual navigation, Nature Reviews Neuroscience, 3 (2002), $542-552$.

[19] J.M. Cornelius, C.W. Breuner, T.P. Hahn, Under a neighbour's influence: public information affects stress hormones and behaviour of a songbird. Proc. R. Soc. B, 277 (2010), 2399-2404. doi: 10.1098/rspb.2010.0164

[20] J.G. Cox and S.L. Lima, Naivete and an aquatic - terrestrial dichotomy in the effects of introduced predators. Trends Ecol. Evol., 21 (2006), No. 12, 674-680.

[21] R. Cross, H. McNamara, A. Pokrovskii, Modelling macroeconomic flows related to large ensembles of elementary exchange operations. Physica B, 403 (2008), 451-455. 
A. Pimenov, T.C. Kelly, A. Korobeinikov, M.J.A. O’Callaghan, A.V. Pokrovskii, D. Rachinskii Memory effects in population dynamics

[22] R. Cross, H. McNamara, A.V. Pokrovskii, D. Rachinskii, A new paradigm for modelling hysteresis in macroeconomic flows. Physica B, 403 (2007), No. 2-3, 231-236.

[23] E. Danchin, L-A. Giraldeau, T.J. Valone, R.H. Wagner, Public Information: From Nosy Neighbours to Cultural Evolution. Science, 305 (2006), 487-491.

[24] Mark Davis, Invasion Biology. Oxford University Press, 2009.

[25] F.B.M. De Waal, Darwin's last laugh. Nature, 460 (2009), 175.

[26] W. Deng, J.B. Aimone, F.H. Gage, New neurons and new memories: how does adult hippocampal neurogenesis affect learning and memory. Nature Reviews Neuroscience, 11 (2010), 229-233.

[27] N.J. Emery, N.S. Clayton, Effects of experience and social context of prospective caching strategies in scrub jays. Nature, 414 (2004), 443-446.

[28] N.J. Emery, J. Dally, N.S. Clayton, Western scrub jays ( Aphelocoma californica) use cognitive strategies to protect their caches from thieving conspecifics. Animal Cognition, 7 (2004), 37-43.

[29] D.H. Everett, W.I. Whitton, A general approach to hysteresis. Transactions of the Faraday Society, 48 (1952), $749-757$.

[30] J.A. Ewing, Experimental research in magnetism. Trans. R. Soc. Lond., 176 (1895), II.

[31] N.M. Ferguson, C.A. Donnelly, R.M. Anderson, The Foot-and-Mouth Epidemic in Great Britain: Pattern of Spread and Impact of Interventions. Science, 292 (2001), No. 5519, 1155-1160.

[32] D. Flynn, O. Rasskazov, On the integration of an ODE involving the derivative of a Preisach nonlinearity. J. Phys. Conf. Ser., 22 (2005), 43-55. doi:10.1088/1742-6596/22/1/003

[33] P. Forterre, The origin of viruses and their possible role in major evolutionary transitions. Virus Research, 117 (2006), $5-16$.

[34] C. Fraser, C.A. Donnelly, S. Cauchemez, W.P. Hanage, M.D.T. Van Kerkhove, D. Hollingsworth, J. Griffin, R.F. Baggaley, H.E. Jenkins, E.J. Lyons, T. Jombart, W.R. Hinsley, N.C. Grassly, F. Balloux, A.C. Ghani, N.M. Ferguson, A. Rambaut, O.G. Pybus, H. Lopez-Gatell, C.M. Alpuche-Aranda, Ietza Bojorquez Chapela, E.P.Zavala, Dulce Ma. Espejo Guevara, F. Checchi, E. Garcia, S. Hugonnet, C. Roth: The WHO Rapid Pandemic Assessment Collaboration. Pandemic Potential of a Strain of Influenza A (H1N1): Early Findings. Science, 324 (2009), 1557-1561.

[35] N. Goldenfeld, C. Woese, Biology's next revolution. Nature, 445 (2007), 369.

[36] W.D. Hamilton, M. Zuk, Heritable true fitness and bright birds: a role for parasites?. Science, 218 (1982), 384-387.

[37] R. Hampton, S.D. Healy, S.J. Shettlewort, A. Kamil, Neurecologists are not made of straw. Trends Cogn. Sci., 6 (2002), $1-2$.

[38] R.D. Hawkins, E. Kandel, C.B. Bailey, Molecular mechanisms of memory storage in Aplysia, Biological Bulletin, 210 (2006), 174-191.

[39] S.D. Healy, S.R. de Kort, N.S. Clayton, The Hippocampus, spatial memory and food hoarding: a puzzle revisited. Trends Ecol. Evol., 20 (2005), 17-22.

[40] J.M. Heffernan, R.J. Smith, L.M. Wahl, Perspectives on the basic reproductive ratio. J. R. Soc. Interface, 2 (2005), No. 4, 281-93. doi:10.1098/rsif.2005.0042

[41] J.M. Hyman, J. Li, Differential susceptibility epidemic models. J. Math. Biol., 50 (2005), No. 62, $626-644$.

[42] L.V. Kalachev, T.C. Kelly, M.J. O'Callaghan, A.V. Pokrovskii, A.A. Pokrovskiy, Analysis of threshold-type behaviour in mathematical models of the intrusion of a novel macroparasite in a host colony. Math. Med. Biol., 28 (2011), No. 4, 287-333. doi: 10.1093/imammb/dqq013

[43] E. Kandel, The Molecular Biology of Memory Storage: A Dialogue between Genes and Synapses. Science, 294 (2001), 1030-1038.

[44] M.J. Keeling, P. Rohani, Modeling Infectious Diseases in Humans and Animals. Princeton University Press, Princeton, 2008.

[45] W.O. Kermack, A.G. McKendrick, A contribution to the mathematical theory of epidemics. Proc. R. Soc. Lond. A, 115 (1927), 700-721.

[46] E.V. Koonin, Darwinian Evolution in the Light of Genomics. Nucleic Acids Research, 37 (2009), $1011-1034$.

[47] E.V. Koonin, Y.I. Wolf, Genomics of Bacteria and Archaea; the Emerging Dynamic View of the Prokaryotic World. Nucleic Acids Research, 36 (2008), 6688-6719.

[48] A. Korobeinikov, Lyapunov functions and global stability for SIR and SIRS epidemiological models with non-linear transmission. Bull. Math. Biol., 68 (2006), 615-626.

[49] A. Korobeinikov, Global properties of infectious disease models with nonlinear incidence. Bull. Math. Biol., 69 (2007), $1871-1886$

[50] A. Korobeinikov, Global asymptotic properties of virus dynamics models with dose dependent parasite reproduction and virulence, and nonlinear incidence rate. Math. Med. Biol., 26 (2009), No. 3, 225-239.

[51] A. Korobeinikov, Stability of ecosystem: global properties of a general predator-prey model. Math. Med. Biol., 26 (2009), 309-321.

[52] A. Korobeinikov, Global properties of a general predator-prey model with non-symmetric attack and consumption rate. Discrete Cont. Dyn.-B, 14 (2010), No. 3, 1095-1103.

[53] A. Korobeinikov, P.K. Maini, Nonlinear incidence and stability of infectious disease models. Math. Med. Biol., 22 (2005), 113-128.

[54] M.A. Krasnosel'skii, A.V. Pokrovskii, Systems with Hysteresis. Nauka, Moscow, 1983 (English edition: Springer, 1989).

[55] P. Krejci, P. O'Kane, A. Pokrovskii, D. Rachinskii, Properties of solutions to a class of differential models incorporating Preisach hysteresis operator. Physica D, in press,(2011). doi:10.1016/j.physd.2011.05.005 
A. Pimenov, T.C. Kelly, A. Korobeinikov, M.J.A. O’Callaghan, A.V. Pokrovskii, D. Rachinskii Memory effects in population dynamics

[56] P. Krejci, P. O'Kane, A. Pokrovskii, D. Rachinskii, Stability results for a soil model with singular hysteretic hydrology. 5th International Workshop on Multi-Rate Processes and Hysteresis (MURPHYS 2010) IOP Publishing, J. Phys. Conf. Ser. 268 (2011) 012016 doi:10.1088/1742-6596/268/1/012016

[57] J. Levenson, J.D. Sweatt, Epigenetic mechanisms in memory formation. Nature Reviews Neuroscience, 6 (2005), $105-117$.

[58] M. Lipsitch, T. Cohen, B. Cooper, J.M. Robins, S. Ma, L. James, G. Gopalakrishna, S.K. Chew, C.C. Tan, M.H. Samore, D. Fisman, M. Murray, Transmission Dynamics and Control of Severe Acute Respiratory Syndrome. Science, 300 (2003), No. 5627, 1966-1970.

[59] E.M. Macphail, J. Bolhuis, The evolution of intelligence: adaptive specialisations versus general process. Biological Reviews, 76 (2001), 341-364.

[60] J.S. Mattick, M.F. Mehler, RNA editing, DNA recoding and the evolution of human cognition. Trends Neurosci., 31 (2008), 227-233.

[61] I.D. Mayergoyz, Mathematical Models for Hysteresis. Springer, New York, 1991.

[62] I.D. Mayergoyz, Mathematical Models of Hysteresis And Their Applications. Elsevier, 2003.

[63] R. Menzel, U. Greggers, A. Smith, S. Berger, S. Brandt, G. Bundrock, T. Plumpe, F. Schaupp, S. Silke, J. Stindt, N. Stollhoff, S. Watzl, Honey bees navigate according to a map-like memory. Proceedings of the National Academy of Sciences, 102 (2006), 3040-3045.

[64] L. Neel, Theories des lois d'aimantation de Lord Rayleigh 1, 2. Cahiers de Physique, 12 (1942), 1-20; 13 (1943), 19-30.

[65] T. Piersma, J.A. van Gils, The Flexible Phenotype A Body - Centered Integration of Ecology, Physiology and Behaviour. Oxford University Press, 2011.

[66] A. Pimenov, Stability and bifurcations of systems with hysteresis and multistable systems. Ph.D. dissertation, University College Cork, Ireland, 2009.

[67] A. Pimenov, D. Rachinskii, Linear Stability Analysis of Systems with Preisach Memory. Discrete Cont. Dyn.-B, 4 (2009), 997-1018.

[68] P. Preisach, Uber die magnetische Nachwirkung. Zeitschrift für Physik, 94 (1935), 277-302.

[69] A. Rodriguez, M. Hausberger, P. Clergeau, Flexibility in European starlings' use of social information: experiments with decoys in different populations. Animal Behaviour, 80 (2010), 965-97. doi:10.1016/ j.anbehav. 2010.08.010

[70] The Science of Hysteresis (Bertotti and Mayergoyz, editors). Vol. 1-3, 125-291, Elsevier Science, 2005. ISBN: 978-012-480874-4.

[71] E. Tulving, Episodic Memory: From Mind to Brain. Ann. Rev. Psychology, 53 (2002), 1-25.

[72] J.J. Templeton, Luc-Alain Giraldea, Vicarious sampling: the use of personal and public information by starlings foraging in a simple patchy environment. Behav Ecol Sociobiol, 38 (1996), 105-114.

[73] A. Visintin, Differential Models of Hysteresis. Springer, Berlin, 1994.

[74] R.H. Wagner, E. Danchin, A taxonomy of biological information. Oikos, 119 (2010), 203-209. doi: 10.1111/j.16000706.2009.17315.x

[75] M.P. Ward, Habitat selection by dispersing yellow-headed blackbirds: evidence of prospecting and the use of public information. Oecologia, 145 (2005), 650-657. doi: 10.1007/s00442-005-0179-0

[76] M. Wonham, M. Lewis, J. Renclawowicz, P. van den Driessche, Transmission assumptions generate conflicting predictions in host-vector disease models: a case study in West Nile virus. Ecology Letters, 9 (2006), 706-725.

[77] X. Yu, T. Tsibane, P.A. McGraw, F.S. House, C.J. Keefer, M.D. Hicar, T.M. Tumpey, C. Pappas, L.A. Perrone, O. Martinez, J. Stevens, I.A. Wilson, P.V. Aguilar, E.L. Altschuler, C.F. Basler, J.E. Crowe Jr, Neutralizing antibodies derived from the $B$ cells of 1918 influenza pandemic survivors. Nature, 445 (2008), 532-536.

[78] M. Zanetti, G. Franchini, T cell memory and protective immunity by vaccination: is more better?. Trends Immunol., 27 (2005), 511-517.

[79] C. Zhao, W. Deng, F.W. Gage, Mechanisms and Functional Implications of Adult Neurogenesis. Cell, 132 (2008), 645-660. 